\title{
CLOSURE-INTERIOR DUALITY OVER COMPLETE LOCAL RINGS
}

\author{
NEIL EPSTEIN AND REBECCA R.G.
}

\begin{abstract}
We define a duality operation connecting closure operations, interior operations, and test ideals, and describe how the duality acts on common constructions such as trace, torsion, tight and integral closures, and divisible submodules. This generalizes the relationship between tight closure and tight interior given in ES14 and allows us to extend commonly used results on tight closure test ideals to operations such as those above.
\end{abstract}

\section{Contents}

1. Introduction

2. Submodule selectors and residual operations 3

3. Duality between submodule selectors 8

4. Closure-interior duality 10

5. Test ideals for preradicals 11

6. Exactness properties for preradicals 14

7. Limits of submodule selectors $\quad 15$

8. Special cases: trace, torsion, completion, and module closures 18

9. Special cases: torsion and divisibility for a multiplicative set 25

10. Special case: tight closure and integral closure 27

11. Special case: almost ring theory, Heitmann closures and post-2016 mixed characteristic closures 29

12. Good localization is dual to good colocalization 31

Acknowledgment 33

References $\quad 34$

\section{INTRODUCTION}

Closure operations have long been an important subject in commutative algebra (c.f. the first named author's survey Eps12]). Many of these closures, including tight closure, are residual, i.e., the closure of a submodule

Date: April 22, 2021.

2010 Mathematics Subject Classification. Primary: 13J10, Secondary: 13A35, 13B22, 13C12, $13 \mathrm{C} 60$.

Key words and phrases. closure operation, test ideal, interior operation, trace, torsion, tight closure, integral closure, Matlis duality, complete local rings. 
$N$ of an $R$-module $M$ is computable from the closure of 0 in $M / N$. Coordinated with this is the study of test ideals, traditionally a part of the study of tight closure theory. In its simplest form, the test ideal is the ideal of ring elements that uniformly kill the closure of zero in every module. In the special case of complete local rings, the tight closure test ideal can be recovered from the Matlis dual to the closure of zero in the injective hull of the residue field. Duals to closures of zero in other modules can then be seen as yielding an interior operation, as detailed in [ES14. The specific case of module closures and trace ideals is discussed in [PR20].

Techniques for analyzing these structures are scattered throughout the literature. In this paper, we prove that this duality holds for all residual closure operations on complete local rings, demonstrate how properties of closures/interiors pass to their dual operations, and show how this framework applies to closures and interiors that appear throughout the literature.

The paper is structured as follows.

Section 2 introduces our most general context of submodule selectors and extensive operations on a category of modules over an associative ring. In it, we give a bijection (see Proposition 2.4) between residual operations on pairs of modules $N \subseteq M$ and submodule selectors on (single) modules. We then detail how properties of submodule selectors correspond to properties of residual operations across this bijection (see Proposition 2.6). This allows us to reduce every aspect of the study of residual closure operations to the study of a certain class of submodule selectors - namely the co-idempotent preradicals.

In Section 3, we restrict our attention to categories of modules over a complete Noetherian local ring that satisfy Matlis duality - a restriction we commonly rely on throughout the paper. In that context, we introduce a duality between submodule selectors (the smile dual), and in Theorem 3.2. we detail how properties of submodule selectors relate across this duality.

Combining these approaches, in Section 4, we construct a duality between residual operations and submodule selectors that restricts to a duality between residual closure operations on the one hand and interior operations on the other. In Theorem 4.3 we prove this, along with an exploration of how several other properties translate across this duality.

Section 5 explores the general notion of test ideals. In it, we show that a version of the theorems describing tight closure test ideals as a submodule of the injective hull of the residue field [HH90] and as a sum of images of maps to $R$ HT04] holds quite generally for residual closure operations arising from preradicals (i.e. functorial submodule selectors) on a category of artinian $R$-modules. See Theorem 5.5 .

Section 6 is devoted to exactness properties on preradicals. We show in Proposition 6.5 that left exactness is dual to surjection-preservation.

In Section 17, we introduce the notions of direct and inverse limits of submodule selectors, and we show (see Propositions 7.2 and 7.4) that many 
good properties are preserved by our duality. This framework becomes useful later, for example in Corollary 8.23. Proposition 9.3, and Proposition 11.3.

The next few sections apply the above framework to various special cases. Section 8 develops the notions of trace and module torsion, both indexed by pairs of modules, as smile-duals to each other (see Theorem 8.18). We show that our general notion of trace behaves well with respect to flat base change (see Theorem 8.12). We relate these notions to module closures (see Remark 8.20), zeroth local cohomology, and $I$-adic completion (see Corollary 8.23). In Proposition 8.25, we find an application of Theorem 5.5 to module closures by finite modules.

Section 9 applies our framework to notions of torsion (with respect to a multiplicative set) and divisibility. Namely, it turns out that the $W$-torsion preradical is smile-dual to the $W$-divisible preradical (see Proposition 9.3, where we also identify the relevant properties of these preradicals).

Section 10 shows how our framework applies to the inspiration for our study, namely tight closure, (liftable) integral closure, and their associated test ideals.

Section 11 connects our framework to some mixed characteristic operations of Pérez and the second named author in [PR20, meant to provide a mixed characteristic version of tight closure theory. Our insistence on indexing both trace and module torsion by a pair, as well as our development of limits of submodule selectors, are particularly relevant here.

Section 12, the final section of our paper, concerns localization and the style of "colocalization" favored by K. Smith and A. Richardson, particularly in relation to smile-duality on systems of submodule selectors. As a result, we show that despite the fact BM10 that tight closure of finite modules does not commute with localization, we have that tight closure in Artinian modules commutes with colocalization (see Theorem [12.4). We use this to shed light on the result of Lyubeznik and Smith [LS01, Theorem 7.1] that formation of the big test ideal commutes with localization and completion (see Corollary 12.5 and its proofs).

\section{Submodule SElectors And Residual operations}

In this section we define and give the basic properties of submodule selectors and residual operations, which will be the fundamental objects of study in this paper. These definitions are inspired by and generalize the notions of closure operation and interior operation. We work as generally as possible in this section, so that we may choose which additional assumptions to work with in later sections.

For the current section, $R$ is an arbitrary ring with identity (not necessarily commutative), and all modules are left $R$-modules.

Definition 2.1. Let $\mathcal{M}$ be a class of $R$-modules that is closed under taking submodules and quotient modules. Let $\mathcal{P}:=\mathcal{P}_{\mathcal{M}}$ denote the set of all pairs $(L, M)$ where $M \in \mathcal{M}$ and $L$ is a submodule of $M$ in $\mathcal{M}$. 
A submodule selector is a function $\alpha: \mathcal{M} \rightarrow \mathcal{M}$ such that

- $\alpha(M) \subseteq M$ for each $M \in \mathcal{M}$, and

- For any isomorphic pair of modules $M, N \in \mathcal{M}$ and any isomorphism $\varphi: M \rightarrow N$, we have $\varphi(\alpha(M))=\alpha(\varphi(M))$.

We say that a submodule selector $\alpha$ is

- order-preserving if for any $(L, M) \in \mathcal{P}$, we have $\alpha(L) \subseteq \alpha(M)$;

- surjection-functorial if for any surjective map $\pi: M \rightarrow N$ in $\mathcal{M}$, we have $\pi(\alpha(M)) \subseteq \alpha(N)$;

- functorial if $\alpha$ is order-preserving and surjection-functorial- i.e. if for any $g: M \rightarrow N$ in $\mathcal{M}$, we have $g(\alpha(M)) \subseteq \alpha(N)$;

- idempotent if for any $M \in \mathcal{M}$ we have $\alpha(\alpha(M))=\alpha(M)$.

- co-idempotent if for any $M \in \mathcal{M}$, we have $\alpha(M / \alpha(M))=0$;

- an interior operation if it is idempotent and order-preserving.

Definition 2.2. An extensive operation on $\mathcal{M}$ is a function $e: \mathcal{P} \rightarrow \mathcal{M}$ such that

- For any $(L, M) \in \mathcal{P}$, we have $L \subseteq e(L, M) \subseteq M$, and

- For any isomorphic pair of modules $M, N \in \mathcal{M}$, any isomorphism $\varphi: M \rightarrow N$, and any submodule $L$ of $M$, we have $\varphi(e(L, M))=$ $e(\varphi(L), N)$.

When $e$ is an extensive operation, we use the standard notation for closure operations $L_{M}^{e}:=e(L, M)$. Hence, the second condition above becomes $\varphi\left(L_{M}^{e}\right)=\varphi(L)_{N}^{e}$.

We say that an extensive operation $e$ on $\mathcal{M}$ is

- residual if for any surjective map $q: M \rightarrow P$ in $\mathcal{M}$, we have $(\operatorname{ker} q)_{M}^{e}=$ $q^{-1}\left(0_{P}^{e}\right)$ (because $q$ is a surjection, we also have $\left.q\left((\operatorname{ker} q)_{M}^{e}\right)=0_{P}^{e}\right)$;

- order-preserving on submodules if whenever $L \subseteq M \subseteq N$ are in $\mathcal{M}$ with $(L, N),(M, N) \in \mathcal{P}$, we have $L_{N}^{e} \subseteq M_{N}^{e}$.

- order-preserving on ambient modules if whenever $L \subseteq M \subseteq N$ are in $\mathcal{M}$ with $(L, M),(L, N) \in \mathcal{P}$, we have $L_{M}^{e} \subseteq L_{N}^{e}$.

- idempotent if for any $(L, M) \in \mathcal{P},\left(L_{M}^{e}\right)_{M}^{e}=L_{M}^{e}$.

- surjection-functorial if whenever $(L, M) \in \mathcal{P}$ and $\pi: M \rightarrow N$ a surjection in $\mathcal{M}$, we have $\pi\left(L_{M}^{e}\right) \subseteq \pi(L)_{N}^{e}$.

- functorial if whenever $(L, M) \in \mathcal{P}$ and $g: M \rightarrow N$ is a map in $\mathcal{M}$, we have $g\left(L_{M}^{e}\right) \subseteq g(L)_{N}^{e}$.

- a closure operation if it is order-preserving on submodules and idempotent.

The residual property will take on special import for us, so that we will use the expression residual operation to mean a residual extensive operation.

Next we construct a map $\rho$ that takes as input a submodule selector and outputs a residual operation, and a map $\sigma$ that takes as input a residual operation and outputs a submodule selector. 
Construction 2.3. If $\alpha$ is a submodule selector on $\mathcal{M}$, define $\rho(\alpha): \mathcal{P} \rightarrow \mathcal{M}$ by

$$
L_{M}^{\rho(\alpha)}:=\pi^{-1}(\alpha(M / L)),
$$

where $\pi: M \rightarrow M / L$ is the natural surjection.

Conversely, let $r$ be a residual operation on $\mathcal{M}$. Define $\sigma(r): \mathcal{M} \rightarrow \mathcal{M}$ by

$$
\sigma(r)(M):=0_{M}^{r}
$$

Proposition 2.4. The function $\sigma$ given in Construction 2.3 gives a bijection between residual operations on $\mathcal{M}$ and submodule selectors on $\mathcal{M}$, with inverse given by $\rho$.

Proof. First we show that if $\alpha$ is a submodule selector on $\mathcal{M}$, then $\rho(\alpha)$ is a residual operation on $\mathcal{M}$. To see that it is an extensive operation, let $L \subseteq M$ be in $\mathcal{M}$ and let $\pi: M \rightarrow M / L$ be the natural surjection. Then $L=\pi^{-1}(0) \subseteq \pi^{-1}(\alpha(M / L))=L_{M}^{\rho(\alpha)} \subseteq \pi^{-1}(M / L)=M$. Moreover, the isomorphism condition on submodule selectors translates properly via $\rho$ to the isomorphism condition on extensive operations. To see that $\rho(\alpha)$ is residual, let $q: M \rightarrow P$ be a surjective map in $\mathcal{M}$. Let $L=\operatorname{ker} q$. Let $j: P \rightarrow M / L$ be the isomorphism such that $\pi:=j \circ q: M \rightarrow M / L$ is the natural surjection. Then

$$
\begin{aligned}
(\operatorname{ker} q)_{M}^{\rho(\alpha)} & =L_{M}^{\rho(\alpha)}=\pi^{-1}(\alpha(M / L)) \\
& =q^{-1}\left(j^{-1}(\alpha(M / L))\right)=q^{-1}\left(j^{-1}(\alpha(j(P)))\right) \\
& =q^{-1}\left(j^{-1}(j(\alpha(P)))\right)=q^{-1}(\alpha(P))=q^{-1}\left(0_{P}^{\rho(\alpha)}\right),
\end{aligned}
$$

where the first equality in the third line follows from the isomorphism condition on submodule selectors. Thus, $\rho(\alpha)$ is a residual operation on $\mathcal{M}$.

Conversely, if $r$ be a residual operation (or any extensive operation) on $\mathcal{M}$, then $\sigma(r)$ is clearly a submodule selector on $\mathcal{M}$.

Finally, we show that these operations are inverses of each other. Accordingly, let $r$ be a residual operation on $\mathcal{M}$. Let $L \subseteq M$ be a submodule inclusion in $\mathcal{M}$, and $\pi: M \rightarrow M / L$ the natural surjection. Then

$$
L_{M}^{\rho(\sigma(r))}=\pi^{-1}(\sigma(r)(M / L))=\pi^{-1}\left(0_{M / L}^{r}\right)=L_{M}^{r},
$$

where the last equality follows from the residual condition. On the other hand, let $\alpha$ be a submodule selector on $\mathcal{M}$. Then

$$
\sigma(\rho(\alpha))(M)=0_{M}^{\rho(\alpha)}=\alpha(M),
$$

since the natural surjection $M \rightarrow M$ is just the identity map.

For the next results, we will find it convenient to use the following general lemma about commutative squares. 
Lemma 2.5. Suppose that the following represents a commutative diagram of sets and functions:

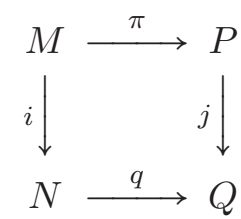

Then for any subset $P^{\prime} \subseteq P, i\left(\pi^{-1}\left(P^{\prime}\right)\right) \subseteq q^{-1}\left(j\left(P^{\prime}\right)\right)$.

Proof. We have $q\left(i\left(\pi^{-1}\left(P^{\prime}\right)\right)\right)=j\left(\pi\left(\pi^{-1}\left(P^{\prime}\right)\right)\right) \subseteq j\left(P^{\prime}\right)$. Applying $q^{-1}$, we get

$$
i\left(\pi^{-1}\left(P^{\prime}\right)\right) \subseteq q^{-1}\left(q\left(i\left(\pi^{-1}\left(P^{\prime}\right)\right)\right)\right) \subseteq q^{-1}\left(j\left(P^{\prime}\right)\right) .
$$

Next we show which properties of submodule selectors and residual operations are preserved by $\rho$ and $\sigma$. In Sections 3 and 4 we will apply this result to our duality between closure operations and interior operations.

Proposition 2.6. Let $\alpha$ be a submodule selector on $\mathcal{M}$ with $r=\rho(\alpha)$ under the bijection given in the previous proposition (so that $\alpha=\sigma(r)$ ). Then:

(1) $\alpha$ is order-preserving if and only if $r$ is order-preserving on ambient modules,

(2) The following are equivalent:

(a) $r$ is order-preserving on submodules,

(b) $\alpha$ is surjection-functorial

(c) $r$ is surjection-functorial,

(3) $\alpha$ is co-idempotent if and only if $r$ is idempotent, and

(4) $\alpha$ is idempotent if and only if $r$ satisfies $L_{L_{M}^{r}}^{r}=L_{M}^{r}$ for all $(L, M) \epsilon$ $\mathcal{P}$.

Proof of (11). Suppose $\alpha$ is order-preserving. Let $L \subseteq M \subseteq N$ be submodule inclusions in $\mathcal{M}$. Let $p: N \rightarrow N / L$ be the canonical surjection, and $\pi$ its restriction to $M \rightarrow M / L$. Then $\alpha(M / L) \subseteq \alpha(N / L)$ by assumption, so we have

$$
L_{M}^{r}=\pi^{-1}(\alpha(M / L))=p^{-1}(\alpha(M / L)) \subseteq p^{-1}(\alpha(N / L))=L_{N}^{r} .
$$

Conversely, suppose $r$ is order-preserving on ambient modules. Let $L \subseteq M$ be a submodule inclusion in $\mathcal{M}$. Then $\alpha(L)=0_{L}^{r} \subseteq 0_{M}^{r}=\alpha(M)$.

Proof of (2). (2a) $\Longrightarrow$ (2b): Suppose $r$ is order-preserving on submodules. Let $\pi: M \rightarrow P$ be a surjection in $\mathcal{M}$. Without loss of generality, $P=M / L$ where $L=\operatorname{ker}(\pi)$ and $\pi$ is canonical. By assumption, we have $0_{M}^{r} \subseteq L_{M}^{r}$. Hence, $\pi(\alpha(M))=\pi\left(0_{M}^{r}\right) \subseteq \pi\left(L_{M}^{r}\right)=\pi\left(\pi^{-1}(\alpha(M / L))\right)=\alpha(M / L)=\alpha(P)$.

$(2 \mathrm{~b}) \Longrightarrow(2 \mathrm{c}):$ Suppose $\alpha$ is surjection-functorial. Let $\pi: M \rightarrow P$ be a surjection in $\mathcal{M}$. Let $(L, M) \in \mathcal{P}$. Then consider the following commutative 
diagram:

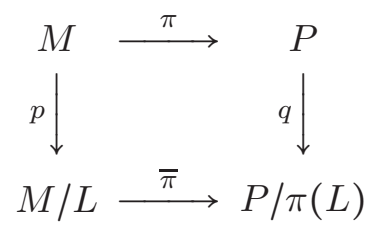

Using Lemma 2.5, we have: $\pi\left(L_{M}^{r}\right)=\pi\left(p^{-1}(\alpha(M / L))\right) \subseteq q^{-1}(\bar{\pi}(\alpha(M / L))) \subseteq$ $q^{-1}(\alpha(P / \pi(L)))=\pi(L)_{P}^{r}$.

$(2 \mathrm{c}) \Longrightarrow$ (2a): Suppose $r$ is surjection-functorial. Let $L \subseteq M \subseteq N$ be submodule inclusions with $(L, N),(M, N) \in \mathcal{P}$. Consider the natural map $q: N \rightarrow N / M$. Then

$$
\frac{L_{N}^{r}+M}{M}=q\left(L_{N}^{r}\right) \subseteq q(L)_{N / M}^{r}=0_{N / M}^{r}=q\left(M_{N}^{r}\right)=\frac{M_{N}^{r}}{M} .
$$

Hence, $L_{N}^{r} \subseteq M_{N}^{r}$.

Proof of (3). Suppose $\alpha$ is co-idempotent. Let $(L, M) \in \mathcal{P}$, and let $q$ denote the natural surjection $M \rightarrow M / L_{M}^{r}$. We have

$$
\begin{aligned}
0 & =\alpha\left(\frac{M / L}{\alpha(M / L)}\right)=\alpha\left(\frac{M / L}{0_{M / L}^{r}}\right)=\alpha\left(\frac{M / L}{\pi\left(L_{M}^{r}\right)}\right)=\alpha\left(\frac{M / L}{L_{M}^{r} / L}\right)=\alpha\left(\frac{M}{L_{M}^{r}}\right) \\
& =q\left(q^{-1}\left(\alpha\left(M / L_{M}^{r}\right)\right)\right)=q\left(\left(L_{M}^{r}\right)_{M}^{r}\right)=\frac{\left(L_{M}^{r}\right)_{M}^{r}}{L_{M}^{r}} .
\end{aligned}
$$

Hence $L_{M}^{r}=\left(L_{M}^{r}\right)_{M}^{r}$, so that $r$ is idempotent.

Conversely suppose $r$ is idempotent. Let $M \in \mathcal{M}$, and let $\pi: M \rightarrow M / 0_{M}^{r}$ be the natural surjection. Then $0_{M}^{r}=\left(0_{M}^{r}\right)_{M}^{r}$, so

$\alpha(M / \alpha(M))=\alpha\left(M / 0_{M}^{r}\right)=\pi\left(\pi^{-1}\left(\alpha\left(M / 0_{M}^{r}\right)\right)\right)=\pi\left(\left(0_{M}^{r}\right)_{M}^{r}\right)=\left(0_{M}^{r}\right)_{M}^{r} / 0_{M}^{r}=0$.

Thus $\alpha$ is co-idempotent.

Proof of [4). We have $\alpha(\alpha(M))=\alpha\left(0_{M}^{r}\right)=0_{0_{M}^{r}}^{r}$,

Suppose $\alpha$ is idempotent. We will show that $L_{L_{M}^{r}}^{r}=L_{M}^{r}$ for all $(L, M) \in \mathcal{P}$. Since $\alpha$ is idempotent, $0_{0_{M}^{r}}^{r}=0_{M}^{r}$ for all $M \in \mathcal{M}$. Let $(L, M) \in \mathcal{P}$. Then $L_{M}^{r}=\pi^{-1}\left(0_{M / L}^{r}\right)$, where $\pi: M \rightarrow M / L$ is the quotient map. So if $p: L_{M}^{r} \rightarrow$ $L_{M}^{r} / L$ is the restriction of $\pi$ to $L_{M}^{r}$, we have

$$
L_{L_{M}^{r}}^{r}=p^{-1}\left(0_{L_{M}^{r} / L}^{r}\right) .
$$

But $L_{M}^{r}=\pi^{-1}\left(0_{M / L}^{r}\right)$. So $L_{M}^{r} / L=\pi\left(L_{M}^{r}\right)=\pi\left(\pi^{-1}\left(0_{M / L}^{r}\right)\right)=0_{M / L}^{r}$, since $\pi$ is a surjection. Hence

$$
L_{L_{M}^{r}}^{r}=p^{-1}\left(0_{L_{M}^{r} / L}^{r}\right)=p^{-1}\left(0_{0_{M / L}^{r}}^{r}\right)=p^{-1}\left(0_{M / L}^{r}\right) .
$$

Since $p$ is the restriction of $\pi$ to $L_{M}^{r}$, this is $\pi^{-1}\left(0_{M / L}^{r}\right) \cap L_{M}^{r}=L_{M}^{r} \cap L_{M}^{r}=L_{M}^{r}$, as desired. 
Now suppose that $L_{L_{M}^{r}}^{r}=L_{M}^{r}$ for all $L \subseteq M$. In particular, we have $0_{0_{M}^{r}}^{r}=0_{M}^{r}$ for each $M \in \mathcal{M}$. This implies that $\alpha$ is idempotent.

\section{Duality Between submodule selectors}

In this section, we define a duality operation $\checkmark$ between submodule selectors and show which properties from Section 2 correspond under this duality. We then give a useful alternate characterization of the duality.

Throughout this section, $R$ is a complete Noetherian local ring with maximal ideal $\mathfrak{m}$, residue field $k$, and $E:=E_{R}(k)$ the injective hull. We will use $\checkmark$ to denote the Matlis duality operation. $\mathcal{M}$ is a category of $R$-modules closed under taking submodule and quotient modules, and such that for all $M \in \mathcal{M}, M^{\vee \vee} \cong M$. So for example $\mathcal{M}$ could be the category of finitely generated $R$-modules, or of Artinian $R$-modules.

Definition 3.1. Let $S(\mathcal{M})$ denote the set of all submodule selectors on $\mathcal{M}$. Define $\cup: S(\mathcal{M}) \rightarrow S\left(\mathcal{M}^{\vee}\right)$ as follows: For $\alpha \in S(\mathcal{M})$ and $M \in \mathcal{M}^{\vee}$,

$$
\alpha^{\smile}(M):=\left(M^{\vee} / \alpha\left(M^{\vee}\right)\right)^{\vee},
$$

considered as a submodule of $M$ in the usual way.

It is clear that the isomorphism-preserving property of $\alpha$ will be inherited by $\alpha^{\leftrightharpoons}$. Moreover, we have the following.

Theorem 3.2. Let $\alpha$ be a submodule selector on $\mathcal{M}$. Then:

(1) $\left(\alpha^{\smile}\right)^{\smile}=\alpha$,

(2) $\alpha$ is surjection-functorial if and only if $\alpha^{\smile}$ is order-preserving, and

(3) $\alpha$ is idempotent if and only if $\alpha^{\smile}$ is co-idempotent.

Proof. First we show that $\alpha^{\sim}=\alpha$. For this, we have

$$
\left(\alpha^{\smile}\right)^{\smile}(M)=\left(M^{\vee} / \alpha^{\smile}\left(M^{\vee}\right)\right)^{\vee}=\left(M^{\vee} /(M / \alpha(M))^{\vee}\right)^{\vee}=\left(\alpha(M)^{\vee}\right)^{\vee}=\alpha(M) .
$$

Next, suppose $\alpha$ is surjection-functorial. Let $L \subseteq M$ be a submodule inclusion in $\mathcal{M}^{\vee}$, and let $\pi: M^{\vee} \rightarrow L^{\vee}$ be the associated surjection in $\mathcal{M}$. Since $\pi\left(\alpha\left(M^{\vee}\right)\right) \subseteq \alpha\left(L^{\vee}\right)$ by assumption on $\alpha$, we have the induced surjections

$$
\frac{M^{\vee}}{\alpha\left(M^{\vee}\right)} \rightarrow \frac{\pi\left(M^{\vee}\right)}{\pi\left(\alpha\left(M^{\vee}\right)\right)}=\frac{L^{\vee}}{\pi\left(\alpha\left(M^{\vee}\right)\right)} \rightarrow \frac{L^{\vee}}{\alpha\left(L^{\vee}\right)}
$$

Dualizing the composite then induces an injective map $\alpha^{\smile}(L) \hookrightarrow \alpha^{\smile}(M)$, and since everything was natural, it follows that $\alpha^{\smile}(L) \subseteq \alpha^{\smile}(M)$.

For the converse, suppose $\beta=\alpha^{\smile}$ is order-preserving. Let $\pi: M \rightarrow P$ be a surjection in $\mathcal{M}$. Without loss of generality, $P=M / L$ and $\pi$ is the canonical surjection, where $L$ is a submodule of $M$. Taking Matlis duals, we have that 
$(M / L)^{\vee}$ is a submodule of $M^{\vee}$. Then

$$
\begin{aligned}
\left(\frac{M}{\pi^{-1}(\alpha(M / L))}\right)^{\vee} & \cong\left(\frac{M / L}{\alpha(M / L)}\right)^{\vee}=\alpha^{\smile}\left((M / L)^{\vee}\right)=\beta\left((M / L)^{\vee}\right) \\
& \subseteq \beta\left(M^{\vee}\right)=\alpha^{\smile}\left(M^{\vee}\right)=\left(\frac{M}{\alpha(M)}\right)^{\vee},
\end{aligned}
$$

where the containment in the second line above arises from the order-preservation property of $\beta$. By naturality of the isomorphism above, it follows that the dual surjection $M / \alpha(M) \rightarrow M / \pi^{-1}(\alpha(M / L))$ is also canonical, so that $\alpha(M) \subseteq \pi^{-1}(\alpha(M / L))$. Applying $\pi$ to both sides, it follows that $\pi(\alpha(M)) \subseteq$ $\pi\left(\pi^{-1}(\alpha(M / L))\right)=\alpha(M / L)$. Thus, $\alpha$ is surjection-functorial.

For (3), first suppose $\alpha$ is idempotent and let $M \in \mathcal{M}^{\vee}$. Then

$$
\alpha^{\smile}\left(M / \alpha^{\smile}(M)\right)=\left(\frac{\alpha\left(M^{\vee}\right)}{\alpha\left(\alpha\left(M^{\vee}\right)\right)}\right)^{\vee}=0^{\vee}=0 .
$$

Finally, suppose $\beta=\alpha^{\smile}$ is co-idempotent. Let $M \in \mathcal{M}$. Then

$$
\begin{aligned}
\alpha(\alpha(M)) & =\beta^{\smile}\left(\beta^{\smile}(M)\right)=\beta^{\smile}\left(\left(\frac{M^{\vee}}{\beta\left(M^{\vee}\right)}\right)^{\vee}\right) \\
& =\left(\frac{M^{\vee} / \beta\left(M^{\vee}\right)}{\beta\left(M^{\vee} / \beta\left(M^{\vee}\right)\right)}\right)^{\vee}=\left(M^{\vee} / \beta\left(M^{\vee}\right)\right)^{\vee} \\
& =\beta^{\smile}(M)=\alpha(M)
\end{aligned}
$$

where the second equality of the second line comes from the fact that $\beta$ is co-idempotent.

Given a submodule selector $\alpha$, we give an alternate description of $\alpha^{\smile}$ that will be useful later on.

Theorem 3.3. Let $\alpha$ be a submodule selector and $M$ an $R$-module. Then

$$
\alpha^{\smile}(M)=\left\{z \in M: g(z)=0 \text { for all } g \in \alpha\left(M^{\vee}\right)\right\}
$$

Proof. We have an exact sequence

$$
0 \rightarrow \alpha\left(M^{\vee}\right) \rightarrow M^{\vee} \rightarrow M^{\vee} / \alpha\left(M^{\vee}\right) \rightarrow 0,
$$

whose Matlis dual is

$$
0 \leftarrow \alpha\left(M^{\vee}\right)^{\vee} \stackrel{q}{\leftarrow} M^{\vee \vee} \leftarrow \alpha^{\smile}(M) \leftarrow 0
$$

Note that $q$ is the restriction map.

Set $i$ to be the standard isomorphism $M \cong M^{\vee \vee}$, and let $\mu: M \rightarrow \alpha\left(M^{\vee}\right)^{\vee}$ be $q \circ i$. This implies that

$$
\alpha^{\smile}(M)=\left(M^{\vee} / \alpha\left(M^{\vee}\right)\right)^{\vee}=\operatorname{ker}\left(M \stackrel{\mu}{\rightarrow}\left(\alpha\left(M^{\vee}\right)\right)^{\vee}\right) \subseteq M .
$$

Note that for $z \in M$ and $g: M \rightarrow E, i(z)(g)=g(z)$. So

$$
(q \circ i)(z)=\left.i(z)\right|_{\alpha\left(M^{\vee}\right)},
$$

where $i(z): M^{\vee} \rightarrow E$. 
An element $z \in \operatorname{ker}(\mu)=\operatorname{ker}(q \circ i)$ if and only if $\left.i(z)\right|_{\alpha\left(M^{\vee}\right)}=0$, if and only if $\alpha\left(M^{\vee}\right) \subseteq \operatorname{ker}(i(z))$, if and only if for every $g \in \alpha\left(M^{\vee}\right), g(z)=0$, if and only if $z \in \bigcap_{g \in \alpha\left(M^{\vee}\right)} \operatorname{ker}(g)$.

\section{Closure-interior Duality}

Using the results of Section [3, we achieve our original goal of a duality between residual closure operations and interior operations such as that between tight closure and tight interior [ES14, and we indicate the properties that correspond via this duality.

In this section, $R$ is a complete local Noetherian ring.

Definition 4.1. If $r$ be a residual operation on $\mathcal{P}\left(\right.$ resp. $\left.\mathcal{P}^{\vee}\right)$, set $i(r):=$ $\sigma(r)^{\smile}$. If $j$ is a submodule selector on $\mathcal{M}$ (resp. $\left.\mathcal{M}^{\vee}\right)$, set $c(j):=\rho\left(j^{\smile}\right)$. Here ${ }^{\sim}$ is as in $\$ 3$ and $\rho, \sigma$ are as in Construction 2.3 .

In the special cases where $r$ is restricted to be a closure operation, or $j$ an interior operation, the above gives the duality we desire, as outlined in Lemma 4.2 and Theorem 4.3 below.

Lemma 4.2. We have that $c$ and $i$ are inverses of one another. Hence, there is a one-to-one correspondence between residual operations on $\mathcal{P}$ and submodule selectors on $\mathcal{M}^{\vee}$.

Proof. This follows from Proposition 2.4 and Theorem 3.2(1).

Theorem 4.3. Let $r$ be a residual operation on $\mathcal{P}$, and $\mathrm{i}:=i(r)$. Then:

(1) $r$ is order-preserving on submodules $\Longleftrightarrow \mathrm{i}$ is order-preserving.

(2) $r$ is idempotent $\Longleftrightarrow \mathrm{i}$ is idempotent.

(3) $r$ is a closure operation $\Longleftrightarrow \mathrm{i}$ is an interior operation.

(4) Suppose $r$ is a closure operation (i.e. i is an interior operation). The following are equivalent:

(a) $r$ is functorial.

(b) $r$ is order-preserving on ambient modules.

(c) $\mathrm{i}$ is surjection-functorial.

(d) i is functorial.

Proof. This follows from Proposition 2.6 and Theorem 3.2. Here are the details:

(1): $r$ is order-preserving on submodules $\Longleftrightarrow \sigma(r)$ is surjection-functorial (by Proposition 2.6 (2)) $\Longleftrightarrow \mathrm{i}$ is order-preserving (by Theorem 3.2).

$(2): r$ is idempotent $\Longleftrightarrow \sigma(r)$ is co-idempotent(by Proposition 2.6(3)) $\Longleftrightarrow \mathrm{i}$ is idempotent (by Theorem $3.2(1 \& 3)$ ).

(3) follows from (1) and (2).

(4): $r$ is functorial $\Longleftrightarrow$ it is order-preserving on ambient modules (by Proposition $2.6(2)) \Longleftrightarrow \sigma(r)$ is order-preserving (by Proposition 2.6 (1)) $\Longleftrightarrow \mathrm{i}$ is surjection-functorial(by Theorem $3.2(2)) \Longleftrightarrow \mathrm{i}$ is functorial (by definition of interior operation). 
Summarizing the correspondences given in the above sections, we have the following chart:

\begin{tabular}{|c|c|c|c|c|}
\hline $\begin{array}{c}\text { residual operation } \\
r \\
\rho(\alpha) \\
c(\beta) \\
\text { order-preserving on submodules/ } \\
\text { surjection-functorial } \\
\text { idempotent } \\
\text { order-preserving on ambient modules } \\
\text { closure operation } \\
\text { functorial closure op. }\end{array}$ & $\stackrel{\sigma}{\longrightarrow}$ & \begin{tabular}{|c|} 
submodule selector \\
$\sigma(r)$ \\
$\alpha$ \\
$\beta^{\smile}$ \\
surjection-functorial \\
\\
co-idempotent \\
order-preserving
\end{tabular} & 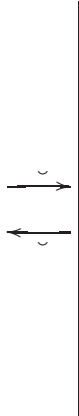 & $\begin{array}{c}\text { submodule selector } \\
\mathrm{i}(r) \\
\alpha- \\
\beta \\
\text { order-preserving } \\
\text { idempotent } \\
\text { surjection-functorial } \\
\text { interior operation } \\
\text { functorial interior op. }\end{array}$ \\
\hline
\end{tabular}

\section{TEST IDEALS FOR PRERADiCALS}

In this section we restrict to the case of preradicals, defined below, and give a more explicit form for the dual of a preradical and its finitistic version. We also give a result (Theorem 5.5) that is a generalization of key properties of the tight closure test ideal originally described in [HH90].

In this section, $R$ will be an arbitrary ring with identity (not necessarily commutative), and all modules are left $R$-modules.

Definition 5.1. (1) A preradical is a functorial submodule selector [DT95].

(2) If $\alpha$ is a submodule selector on $\mathcal{M}$, then the finitistic version $\alpha_{f}$ is the submodule selector on $\mathcal{M}$ given for each $M \in \mathcal{M}$ by

$$
\alpha_{f}(M):=\sum\{\alpha(L) \mid L \subseteq M, L \text { finitely generated, } L \in \mathcal{M}\} .
$$

We present the finitistic version $\alpha_{f}$ of $\alpha$ as a generalization of notions of finitistic tight closure and test ideals.

Lemma 5.2. Let $\alpha$ be a submodule selector on $\mathcal{M}$. Then:

(1) $\alpha_{f}$ is order-preserving,

(2) if $\alpha$ is order-preserving, then $\alpha(M)=\alpha_{f}(M)$ for all finitely generated $M \in \mathcal{M}$, and

(3) if $\alpha$ is surjection-functorial, then $\alpha_{f}$ is a preradical on $\mathcal{M}$.

Proof. Let $L \subseteq M$ be a submodule inclusion in $\mathcal{M}$. Let $x \in \alpha_{f}(L)$. Then there is some finitely generated submodule $L^{\prime}$ of $L$ (hence also of $M$ ) with $L^{\prime} \in \mathcal{M}$ and $x \in \alpha\left(L^{\prime}\right)$. Thus, $x \in \alpha_{f}(M)$.

Now suppose $\alpha$ is order-preserving and let $M \in \mathcal{M}$ be finitely generated. It is clear that $\alpha(M) \subseteq \alpha_{f}(M)$ since $M$ is a finitely generated submodule of $M$ in $\mathcal{M}$. For the other containment, let $x \in \alpha_{f}(M)$. Then there is a finitely generated submodule $L$ of $M$ with $x \in \alpha(L)$. But $\alpha(L) \subseteq \alpha(M)$ since $\alpha$ is order-preserving. So $x \in \alpha(M)$.

Now suppose $\alpha$ is surjection-functorial. Let $g: M \rightarrow N$ be a module map in $\mathcal{M}$. Let $x \in \alpha_{f}(M)$. Then there is a finitely generated submodule $M^{\prime} \subseteq M$, 
$M^{\prime} \in \mathcal{M}$, with $x \in \alpha\left(M^{\prime}\right)$. Let $N^{\prime}:=g\left(M^{\prime}\right)$, and let $g^{\prime}: M^{\prime} \rightarrow N^{\prime}$ be the induced map. Then since $\alpha$ is surjection-functorial, $g(x)=g^{\prime}(x) \in \alpha\left(N^{\prime}\right)$. Since $N^{\prime} \in \mathcal{M}$ and $N^{\prime}$ is a finitely generated submodule of $N$, we have $g(x) \in \alpha_{f}(N)$.

Lemma 5.3. Let $M, N \in \mathcal{M}$ and let $\alpha$ be a preradical on $\mathcal{M}$. Then $\alpha(M \oplus$ $N)=\alpha(M) \oplus \alpha(N)$ as submodules of $M \oplus N$.

Proof. Let $(x, y) \in \alpha(M \oplus N)$. Let $\pi_{1}, \pi_{2}$ be the canonical projections of $M \oplus N$ onto $M, N$ respectively. Then since $\alpha$ is a preradical, $x=\pi_{1}(x, y) \epsilon$ $\alpha(M)$ and $y=\pi_{2}(x, y) \in \alpha(N)$. Thus, $(x, y) \in \alpha(M) \oplus \alpha(N)$.

Conversely, let $(x, y) \in \alpha(M) \oplus \alpha(N)$. Let $i_{1}, i_{2}$ be the canonical injections of $M, N$ respectively into $M \oplus N$. Then since $\alpha$ is a preradical, $(x, 0)=i_{1}(x) \epsilon$ $\alpha(M \oplus N)$ and $(0, y)=i_{2}(y) \in \alpha(M \oplus N)$. Hence $(x, y)=(x, 0)+(0, y) \epsilon$ $\alpha(M \oplus N)$.

The following result was already known (see, for example [Die10]), but we include it to show how it follows naturally from the results of this paper.

Corollary 5.4. Let cl be a residual functorial closure operation on $\mathcal{P}$. Let $U \subseteq L, V \subseteq M$ be submodule inclusions in $\mathcal{P}$. Then

$$
U_{L}^{\mathrm{cl}} \oplus V_{M}^{\mathrm{cl}}=(U \oplus V)_{L \oplus M}^{\mathrm{cl}} .
$$

Proof. Let $\alpha:=\sigma(\mathrm{cl})$. Then by Lemma 5.3, $\alpha((L / U) \oplus(M / V))=\alpha(L / U) \oplus$ $\alpha(M / V)$. Hence, using the standard identification $\frac{L}{U} \oplus \frac{M}{V}=\frac{L \oplus M}{U \oplus V}$, we have

$$
\begin{aligned}
U_{L}^{\mathrm{cl}} \oplus V_{M}^{\mathrm{cl}} & =\pi_{1}^{-1}(\alpha(L / U)) \oplus \pi_{2}^{-1}(\alpha(M / V)) \\
& =\pi^{-1}(\alpha(L / U) \oplus \alpha(M / V)) \\
& =\pi^{-1}(\alpha(L / U \oplus M / V))=\pi^{-1}\left(\alpha\left(\frac{L \oplus M}{U \oplus V}\right)\right) \\
& =(U \oplus V)_{L \oplus M}^{\mathrm{cl}},
\end{aligned}
$$

where $\pi: L \oplus M \rightarrow(L \oplus M) /(U \oplus V)$ is the quotient map, and $\pi_{1}: L \rightarrow L / U$ and $\pi_{2}: M \rightarrow M / V$ are its component maps.

The following result was inspired by the main results of [PR20]. Our result requires the ring to be complete and restricts the module category, but allows arbitrary preradicals (rather than just those coming from module closures). In the case of tight closure, this is a classical result, originally given as Proposition 8.23 in HH90 for the finitistic version and discussed in the lecture notes of October 26th in [Hoc07.

Theorem 5.5. Let $(R, \mathfrak{m}, k)$ be a commutative complete Noetherian local ring and $E$ the injective hull of its residue field. Let $\alpha$ be a preradical on the 
category of Artinian $R$-modules $\mathcal{A}$. Let $\alpha_{f}$ be the finitistic version. Then:

$$
\begin{aligned}
\alpha^{\smile}(R) & =\operatorname{ann}(\alpha(E))=\bigcap_{M \in \mathcal{A}} \operatorname{ann}(\alpha(M)) \\
& \subseteq \alpha_{f}(R)=\operatorname{ann}\left(\alpha_{f}(E)\right) \\
& =\bigcap_{\lambda(M)<\infty} \operatorname{ann}(\alpha(M)) \subseteq \bigcap_{\lambda(R / I)<\infty} \operatorname{ann}(\alpha(R / I)) .
\end{aligned}
$$

Moreover, the last containment is an equality whenever $R$ is approximately Gorenstein (e.g. reduced, or depth at least 2).

If $\alpha$ represents a residual closure operation $\mathrm{cl}$ defined on artinian modules and on all ideals, in such a way that for any ideal $J$, there is a collection $\left\{I_{\lambda}\right\}_{\lambda \in \Lambda}$ of $\mathfrak{m}$-primary ideals for some index set $\Lambda$, such that $J=\bigcap_{\lambda} I_{\lambda}$ and $J^{\mathrm{cl}}=\bigcap_{\lambda} I_{\lambda}^{\mathrm{cl}}$. Then $\bigcap_{\lambda(R / I)<\infty} \operatorname{ann}(\alpha(R / I))=\bigcap_{I \text { ideal of } R}\left(I: I^{\mathrm{cl}}\right)$.

Proof. We prove all the required containments.

To see that $\alpha^{\smile}(R)=\operatorname{ann}(\alpha(E))$, recall that under the identification $R=$ $E^{\vee}$, the dual of the exact sequence $0 \rightarrow \alpha(E) \rightarrow E \rightarrow E / \alpha(E) \rightarrow 0$ becomes $0 \rightarrow(E / \alpha(E))^{\vee} \rightarrow R \stackrel{\pi}{\rightarrow} \alpha(E)^{\vee} \rightarrow 0$, where $\pi(r)(x):=r x$. Thus, we have $\alpha^{\smile}(R)=(E / \alpha(E))^{\vee}=\operatorname{ker} \pi=\{r \in R \mid r x=0$ for all $x \in \alpha(E)\}=\operatorname{ann}(\alpha(E))$.

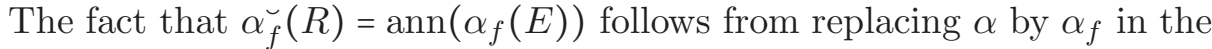
above, using the fact from Lemma 5.2 that $\alpha_{f}$ is a preradical.

To prove that $\operatorname{ann}(\alpha(E)) \subseteq \bigcap_{M \in \mathcal{A}} \operatorname{ann}(\alpha(M))$, we show that $\operatorname{ann}(\alpha(E))$ annihilates $\alpha(M)$ for all artinian $M$. Note that for any $\operatorname{artinian} M$, we have that $M^{\vee}$ is finitely generated. Hence, there is a surjection $p: R^{\oplus t} \rightarrow$ $M^{\vee}$ for some positive integer $t$. Dualizing, we have $p^{\vee}: M \hookrightarrow E^{\oplus t}$. Now, $\operatorname{ann}(\alpha(E))=\operatorname{ann}\left(\alpha(E)^{\oplus t}\right)=\operatorname{ann}\left(\alpha\left(E^{\oplus t}\right)\right)$ by Lemma 5.3. But since $M$ embeds as a submodule of $E^{\oplus t}$ and $\alpha$ is order-preserving, we have $\alpha(M) \hookrightarrow$ $\alpha\left(E^{\oplus t}\right)$. So any element of $R$ that annihilates $\alpha\left(E^{\oplus t}\right)$ annihilates $\alpha(M)$ as well.

The reverse containment $\operatorname{ann}(\alpha(E)) \supseteq \bigcap_{M \in \mathcal{A}}$ ann $(\alpha(M))$ follows from the fact that $E$ is an artinian $R$-module.

Next we show that $\alpha_{f}(E)$ is the common annihilator of all modules $\alpha(M)$ for $M$ finite length. For this, let $r \in \operatorname{ann}\left(\alpha_{f}(E)\right)$. Then for any finite-length $R$-module $M$, we have that $M$ is artinian and $\alpha(M)=\alpha_{f}(M)$. Hence by the above, $r \in \operatorname{ann}(\alpha(M))$. Conversely, let $r \in \bigcap_{\lambda(M)<\infty} \operatorname{ann}(\alpha(M))$. Let $x \in \alpha_{f}(E)$. Then there is a finitely generated (hence finite length) submodule $M$ of $E$ with $x \in \alpha(M)$. Thus $r x=0$, completing the proof of the current claim.

Next we prove that $\alpha^{\smile}(R) \subseteq \alpha_{f}^{\smile}(R)$. For any $r \in \alpha^{\smile}(R)$ and any finite-

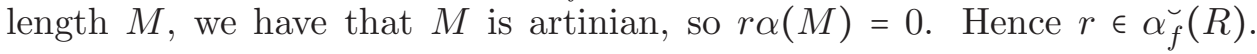

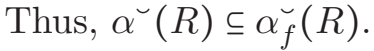

The last displayed containment is clear.

Now suppose $R$ is approximately Gorenstein. Let $M$ be a finite length $R$-module. By Lemma [5.3, we may assume $M$ is $\oplus$-indecomposable. Let $J=\operatorname{ann}(M)$. Since $J$ is $\mathfrak{m}$-primary and $R$ is approximately Gorenstein, 
there is an irreducible $\mathfrak{m}$-primary ideal $I$ with $I \subseteq J$. Then $M$ is a direct sum of indecomposable finite-length modules over the Artinian Gorenstein ring $R / I$. Hence $M \hookrightarrow E_{R / I}(k)=R / I$.

For the final statement, let cl be a closure operation satisfying the given conditions. The containment $\supseteq$ follows from the fact that $\alpha(R / I)=\left(I: I^{\mathrm{cl}}\right)$ for any ideal $I$ of finite colength. Conversely, let $r \in R$ such that $r$ annihilates $\alpha(R / I)$ for every ideal $I$ of finite colength. Let $J$ be an arbitrary ideal and $x \in J^{\mathrm{cl}}$. Let $\left\{I_{\lambda}\right\}_{\lambda \in \Lambda}$ be a collection of ideals as in the hypothesis. Then suppose $r \in \alpha(R / I)$ for all $\mathfrak{m}$-primary $I$. Then for any $x \in J^{\mathrm{cl}}$, we have $x \in I_{\lambda}^{\text {cl }}$ for all $\lambda$, whence $r x \in \bigcap_{\lambda} I_{\lambda}=J$.

Remark 5.6. Recall that the last condition above holds for both tight closure and (liftable) integral closure. Namely, $I^{*}=\bigcap_{n}\left(I+\mathfrak{m}^{n}\right)^{*}$ Hun96, Theorem 1.5(4)] and $I^{-}=\bigcap_{n}\left(I+\mathfrak{m}^{n}\right)^{-}$[HS06, Corollary 6.8.5] respectively.

\section{EXACTNESS PROPERTIES FOR PRERADICALS}

Since a preradical is an additive functor (see Lemma [5.3), it is natural to ask when it preserves exactness. Of course injectivity is preserved by definition. Accordingly, we recall the following results, for which $R$ will be an arbitrary ring with identity (not necessarily commutative), and all modules left $R$-modules.

Definition/Proposition 6.1 (Hereditary and cohereditary preradicals CLVW06, 6.9]). Let $\alpha$ be a preradical on $\mathcal{M}$. Then the following are equivalent

(1) $\alpha$ is left exact.

(2) For any submodule inclusion $L \subseteq M$ in $\mathcal{M}$, we have $\alpha(L)=L \cap \alpha(M)$.

(3) $\alpha$ is idempotent and whenever $L \subseteq M$ is a submodule inclusion in $\mathcal{M}$ with $\alpha(M)=M$, we have $\alpha(L)=L$.

In this case, we say that $\alpha$ is hereditary.

On the other hand, the following are equivalent:

(1) a preserves surjections, i.e. given a surjection $\pi: M \rightarrow N$ in $\mathcal{M}$, $\pi(\alpha(M))=\alpha(N)$.

(2) For any submodule inclusion $L \subseteq M$ in $\mathcal{M}$, we have $\alpha(M / L)=$ $\frac{L+\alpha(M)}{L}$.

(3) $\alpha$ is co-idempotent and whenever $L \subseteq M$ is a submodule inclusion in $\mathcal{M}$ with $\alpha(M)=0$, we have $\alpha(M / L)=0$ as well.

In this case, we say that $\alpha$ is cohereditary.

Remark 6.2. The identity preradical is both hereditary and cohereditary. The same holds for the preradical that sends every module to its zero submodule.

Note also that hereditary is equivalent to left exact, but cohereditary is weaker than right exact.

Corollary 6.3. Let $\alpha$ be a preradical on $\mathcal{M}$. Then $\alpha$ is an exact functor if and only if it is hereditary and cohereditary. 
Remark 6.4. From the point of view of closure operations, such preradicals appear quite scarce. Indeed, both hereditariness and cohereditariness fail for many common closure operations, such as tight closure and liftable integral closure. However, as we see in the next result, these two properties are dual to each other:

Proposition 6.5. Let $R$ be a commutative complete local Noetherian ring and let $\alpha$ be a preradical on $\mathcal{M}^{\vee}$. Then $\alpha^{\smile}$ is hereditary if and only if $\alpha$ is cohereditary.

Proof. Let $L \subseteq M$ be a submodule inclusion. Then $\alpha^{\smile}(M) \cap L=\alpha^{\smile}(L)$ if and only if the induced map $L / \alpha^{\smile}(L) \rightarrow M / \alpha^{\smile}(M)$ is injective. Taking Matlis duals, the above map is injective if and only if the map

$$
\alpha\left(L^{\vee}\right)=\alpha^{\smile \smile}\left(L^{\vee}\right)=\left(L / \alpha^{\smile}(L)\right)^{\vee} \leftarrow\left(M / \alpha^{\smile}(M)\right)^{\vee}=\alpha\left(M^{\vee}\right)
$$

is surjective. But every surjection in $\mathcal{M}^{\vee}$ occurs as a map of the form $M^{\vee} \rightarrow L^{\vee}$, where $L \subseteq M$ is a submodule inclusion in $\mathcal{M}$.

Example 6.6. The casual reader might think that the duality ${ }^{`}$ we are using makes left exactness dual to right exactness. But this is not true, as the following class of examples shows.

Let $I$ be an ideal of a commutative complete local Noetherian ring, and let $\sigma(M):=\operatorname{ann}_{M}(I)$ for all $M \in \mathcal{M}$. Let $\tau=\sigma^{\sim}$; then $\tau(N)=I N$ for all $N \in \mathcal{M}^{\vee}$. We will explore these preradicals further in 88 , It is easily seen both that $\sigma$ is left exact and that $\tau=\sigma^{\smile}$ is not right exact (though it is surjection-preserving). Indeed, it is not even exact in the middle. For a counterexample, take any submodule inclusion $L \subseteq M$ with $I L \neq I M \cap L$.

\section{Limits OF SUBMODULE SELECTORS}

We begin the current section by imposing a binary relation $\leq$ on the collection of all submodule selectors on $\mathcal{M}$, as follows: We say $\alpha \leq \beta$ if $\alpha(M) \subseteq \beta(M)$ for all $M \in \mathcal{M}$. This is easily seen to be a partial order. We will discuss direct and inverse limits of appropriate posets of submodule selectors, and show that the dual operation reverses the type of limit.

In this section, let $R$ be a ring with identity (not necessarily commutative), and all modules are left $R$-modules.

Definition 7.1. Let $\Gamma$ be a directed poset (i.e., for all $i, j \in \Gamma$, there is a $k \in \Gamma$ such that $i, j \leq k)$, and $\left\{s_{j}\right\}_{j \in \Gamma}$ a set of submodule selectors such that if $i \leq j, s_{i} \leq s_{j}$. Define $\left.\underset{j \in \Gamma}{\left(\lim _{j}\right.} s_{j}\right)(M):=\bigcup_{j \in \Gamma} s_{j}(M)=\sum_{j \in \Gamma} s_{j}(M)$.

Proposition 7.2. $s:=\underset{j \in \Gamma}{\lim _{\vec{C}}} s_{j}$ is a submodule selector. Moreover, it is orderpreserving (resp. surjection-functorial, resp. functorial) if all the $s_{j}$ have the corresponding property. Further, assuming that all the $s_{j}$ are functorial, $s$ is hereditary if all the $s_{j}$ are too, and if the $s_{j}$ are cohereditary, then $s$ is also cohereditary, and in particular co-idempotent. 
Proof. To see that $s$ is a submodule selector, let $g: M \rightarrow N$ be an isomorphism in $\mathcal{M}$, and let $x \in s(M)$. Then there is some $j \in \Gamma$ such that $x \in s_{j}(M)$. Since $g$ is an isomorphism and $s_{j}$ is a submodule selector, $g(x) \in s_{j}(N)$. Hence, $g(x) \in \bigcup_{i} s_{i}(N)=s(N)$.

Now suppose all the $s_{j}$ are order-preserving. Let $L \subseteq M$ be a submodule inclusion in $\mathcal{M}$. Let $x \in s(L)$. Then there is some $j$ with $x \in s_{j}(L)$. Since $s_{j}$ is order-preserving, $x \in s_{j}(M)$. Hence $x \in s(M)$.

Next suppose all the $s_{j}$ are surjection-functorial. Let $\pi: L \rightarrow M$ be a surjection in $\mathcal{M}$. Let $x \in s(L)$. Then there is some $j$ with $x \in s_{j}(L)$. Since $s_{j}$ is surjection-functorial, $\pi(x) \in s_{j}(M)$. Hence $\pi(x) \in s(M)$.

Now suppose all the $s_{j}$ are functorial and hereditary. Let $L \subseteq M$ be a submodule inclusion in $\mathcal{M}$. Let $x \in s(M) \cap L$. Then there is some $j$ with $x \in s_{j}(M) \cap L=s_{j}(L)$ (the latter since $s_{j}$ is hereditary), whence $x \in s(L)$.

Suppose the $s_{j}$ are cohereditary. Then in particular they are co-idempotent (see Definition/Proposition 6.1). Let $L \subseteq M$ be a submodule inclusion in $\mathcal{M}$. We first prove that $s$ is co-idempotent.

We have

$$
s(M / s(M))=\sum_{j} s_{j}\left(\frac{M}{\sum_{j} s_{j}(M)}\right) .
$$

Since the $s_{j}$ are co-idempotent, $s_{j}\left(M / s_{j}(M)\right)=0$ for all $j \in \Gamma$. Since the $s_{j}$ are cohereditary, this implies that

$$
s_{j}\left(\frac{M}{\sum_{j} s_{j}(M)}\right)=0
$$

for all $j \in \Gamma$. This implies that their sum is 0 , and so $s(M / s(M))=0$.

Now we prove that if $s(M)=0$, then $s(M / L)=0$. We have

$$
s(M / L)=\sum_{j} s_{j}(M / L)=0,
$$

since $0=s(M)=\sum_{j} s_{j}(M)$ and the $s_{j}$ are cohereditary. Hence $s$ is cohereditary.

Definition 7.3. Let $\Omega$ be an inverse poset and $\left\{t_{j}\right\}_{j \in \Omega}$ a set of submodule selectors on $\mathcal{M}$ such that if $i \leq j$, then $t_{i} \leq t_{j}$. Define $\underset{j \in \Omega}{\lim } t_{j}$ by

$$
\left(\lim _{j} t_{j}\right)(M)=\bigcap_{j \in \Omega} t_{j}(M) .
$$

Proposition 7.4. $t:=\lim _{j \in \Omega} t_{j}$ is a submodule selector. Moreover, it is orderpreserving (resp. surjection-functorial, resp. functorial, resp. hereditary) if all the $t_{j}$ have the corresponding property.

Proof. To see that $t$ is a submodule selector (resp. order-preserving, resp. surjection-functorial) when all the $t_{j}$ have the corresponding property, let $g: L \rightarrow M$ be an isomorphism (resp. injection, resp surjection) in $\mathcal{M}$. Let 
$x \in t(L)$. Then for all $j, x \in t_{j}(L)$. By the given property for all the $t_{j}$ and for $g$, we have $g(x) \in t_{j}(M)$ for all $j$. Hence $g(x) \in \bigcap_{j} t_{j}(M)=t(M)$.

Suppose all of the $t_{j}$ are hereditary, i.e. for any submodule inclusion $L \subseteq M$ in $\mathcal{M}, t_{j}(L)=t_{j}(M) \cap L$. Then

$$
t(L)=\bigcap_{j} t_{j}(L)=\bigcap_{j}\left(t_{j}(M) \cap L\right)=\left(\bigcap_{j} t_{j}(M)\right) \cap L=t(M) \cap L,
$$

so $t$ is also hereditary.

Next, we interface with our notion of duality. For the rest of this section, $R$ is a commutative complete local Noetherian ring.

Proposition 7.5. Let $\alpha, \beta$ be submodule selectors on $\mathcal{M}$ with $\alpha \leq \beta$. Then $\alpha^{\smile} \geq \beta^{\smile}$.

Proof. Let $M \in \mathcal{M}^{\vee}$. Then $\alpha\left(M^{\vee}\right) \subseteq \beta\left(M^{\vee}\right)$, so that $M^{\vee} / \alpha\left(M^{\vee}\right) \rightarrow M^{\vee} / \beta\left(M^{\vee}\right)$. Applying Matlis duality, it follows that $\beta^{\smile}(M)=\left(M^{\vee} / \beta\left(M^{\vee}\right)\right)^{\vee} \subseteq\left(M^{\vee} / \alpha\left(M^{\vee}\right)\right)^{\vee}=$ $\alpha^{\smile}(M)$.

Definition 7.6. If $\Gamma$ is a poset, let $\Gamma^{\prime}$ denote its poset dual.

Proposition 7.7. Let $\Gamma$ be a directed poset and $\left\{s_{j}\right\}_{j \in \Gamma}$ a directed system

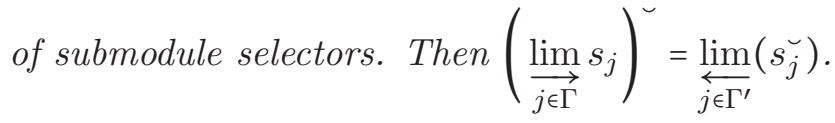

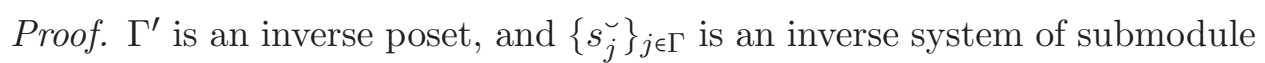

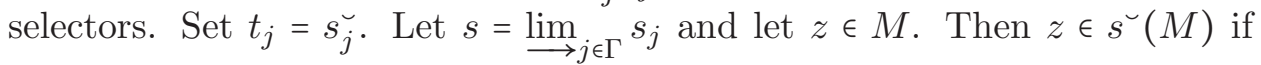
and only if for all $g \in s\left(M^{\vee}\right), z \in \operatorname{ker}(g)$. This holds if and only if for all

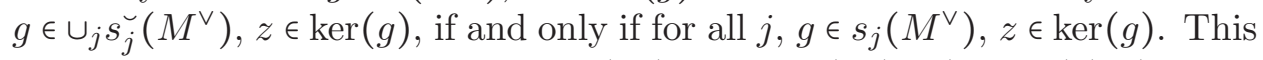

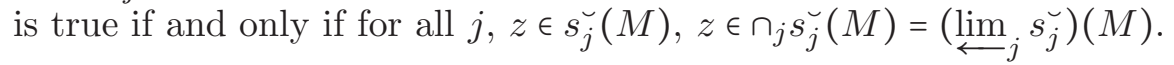

Proposition 7.8. Let $\Omega$ be an inverse poset and $\left\{t_{j}\right\}_{j \in \Omega}$ an inverse system of submodule selectors. Then $\left(\underset{j}{\lim } t_{j}\right)^{\smile}=\underset{j \in \Omega^{\prime}}{\lim }\left(t_{j}^{\longleftarrow}\right)$.

Proof. Write $s_{j}=t_{j}^{\longleftarrow}$. Then $\left\{s_{j}\right\}_{j \in \Omega^{\prime}}$ form a direct limit system. So $\left({\underset{\lim }{j}}_{j} t_{j}\right)^{\smile=}$ $\left(\lim _{j}\left(s_{j}^{\smile}\right)\right)^{\smile}$. By Proposition [7.7, this is equal to $\left(\left(\lim _{\longrightarrow} s_{j}\right)^{\smile}\right)^{\smile}=\underline{\lim }_{\mathfrak{l}} s_{j}=$

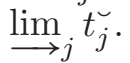

Note that if $N$ is an $R$-module and $\left\{N_{j}\right\}_{j \in \Gamma}$ is a directed system of $R$ modules such that $\lim _{\longrightarrow} N_{j}=N$, it is not always the case that $\lim _{\longleftarrow} t_{N_{j}}=t_{N}$ when $t$ is the trace map defined in Section 8 . So there are examples where

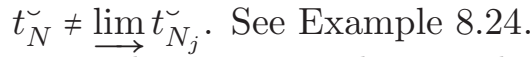

We do get one inclusion when $t$ is trace: $t_{N} \leq \lim _{\leftarrow} t_{N_{j}}$. See Proposition 8.10 . 


\section{Special Cases: trace, torsion, COMpletion, And module CLOSURES}

In this section we apply the structure of the preceding sections to traces, torsion submodules, and module closures, all examples of preradicals and residual operations that appear elsewhere in the literature. The results on the trace are inspired by work of the second named author in [PR20].

Traces. Let $R$ be a commutative ring with identity.

Definition 8.1. Let $L$ be an $R$-module. Then the $L$-trace of an $R$-module $N$ is

$$
\operatorname{tr}_{L}(N)=\operatorname{im}\left(L \otimes \operatorname{Hom}_{R}(L, N) \rightarrow N\right),
$$

where $\ell \otimes f \mapsto f(\ell)$. Equivalently, $\operatorname{tr}_{L}(N)$ is the submodule of $N$ generated by the set

$$
\{f(\ell) \mid f \in \operatorname{Hom}(L, N), \ell \in L\},
$$

or

$$
\operatorname{tr}_{L}(N)=\sum_{f \in \operatorname{Hom}_{R}(L, N)} f(L) .
$$

When $N=R$, this is known as the trace ideal of $L$ [Lam99, Lin17].

Given a subset $S \subseteq L$, the $(S, L)$-trace $\operatorname{tr}_{S, L}(N)$ of $N$ is the $R$-submodule of $N$ generated by the set

$$
\{f(s) \mid f \in \operatorname{Hom}(L, N), s \in S\} .
$$

That is,

$$
\operatorname{tr}_{S, L}(N)=\sum_{f \in \operatorname{Hom}_{R}(L, N)} R f(S) .
$$

Hence, $\operatorname{tr}_{L}=\operatorname{tr}_{L, L}$. If $S=\{x\}$ is a singleton, we write $\operatorname{tr}_{x, L}:=\operatorname{tr}_{\{x\}, L}$.

The ideal $\operatorname{tr}_{x, L}(R)$ is known as the order ideal of $x \in L$ EG89.

If the ring need be specified, we note it in the superscript, as follows:

$$
\operatorname{tr}_{X, L}^{(R)}
$$

Remark 8.2. We note the following easy facts.

(1) For any $R$-module $L$ and any subset $S \subseteq L, \operatorname{tr}_{S, L}$ is a submodule selector on $\mathcal{M}$. In particular, $\operatorname{tr}_{x, L}$ and $\operatorname{tr}_{L}=\operatorname{tr}_{L, L}$ are submodule selectors.

(2) When $L=A$ is an $R$-algebra, $\operatorname{tr}_{A}=\operatorname{tr}_{1, A}$.

(3) When $I$ is an ideal of $R, \operatorname{tr}_{R / I}(N)=\left(0:_{N} I\right)$.

(4) It is elementary that for any $z \in N, R z \cong R / \operatorname{ann}_{R}(z)$. Hence by (3), $\operatorname{tr}_{R z}(N)=\operatorname{ann}_{N}\left(\operatorname{ann}_{R}(z)\right)$.

(5) When $I$ is an ideal of $R$ and $L, N$ are $R$-modules, we have $\operatorname{tr}_{I L, L}(N)=$ $I \operatorname{tr}_{L}(N)$. In particular, $\operatorname{tr}_{I, R}(N)=I N$.

(6) If $L$ is an $R$-module and $x \in L$, then $\operatorname{tr}_{x, L} \leq \operatorname{tr}_{R x}$ : if $z \in \operatorname{tr}_{x, L}(M)$, then there is some $R$-linear $g: L \rightarrow M$ with $g(x)=z$; composing this with the inclusion $i: R x \rightarrow M$, we have $z=(g \circ i)(x)$, so that $z \in \operatorname{tr}_{R x}(M)$. 
(7) However, it is not always the case that $\operatorname{tr}_{R x} \leq \operatorname{tr}_{x, L}$. Let $R=M=$ $k[t]], x=1$, and $L=k((t))$, the fraction field of $R$. Then since $\operatorname{Hom}_{R}(L, M)=0$, we have $\operatorname{tr}_{x, L}(M)=0$. But since $R x$ is a free $R$-module, we have $\operatorname{tr}_{R x}(M)=M$.

Lemma 8.3. Let $L$ be an $R$-module, $S \subseteq L$ a subset, and $R S$ the submodule of $L$ generated by $S$. Then:

(a) $\operatorname{tr}_{S, L}=\operatorname{tr}_{R S, L}$, and

(b) Given $M \in \mathcal{M}$ and $z \in M$, we have $z \in \operatorname{tr}_{S, L}(M)$ if and only if there exists a positive integer $k, R$-linear maps $g_{i}: L \rightarrow M$, and elements $s_{i} \in S$ such that $z=\sum_{i=1}^{k} g_{i}\left(s_{i}\right)$.

Proof. Since $S \subseteq R S, \operatorname{tr}_{S, L} \leq \operatorname{tr}_{R S, L}$. Let $z \in \operatorname{tr}_{R S, L}(M)$. Then there exist $g_{i}: L \rightarrow M$, elements $z_{i} \in R S$, and $r_{i} \in R$ such that $z=\sum_{i} r_{i} g_{i}\left(z_{i}\right)$. Each $z_{i}=\sum_{j} a_{i j} s_{j}$ where the $s_{j} \in S$. Hence

$$
z=\sum_{i, j} a_{i j} r_{i} g_{i}\left(s_{j}\right) \in \operatorname{tr}_{S, L}(M) .
$$

For (b), the "if" direction holds by definition. Conversely, let $z \in \operatorname{tr}_{S, L}(M)$. Then there exist $r_{i} \in R, h_{i}: L \rightarrow M R$-linear, and $s_{i} \in S$ such that $z=$ $\sum_{i=1}^{k} r_{i} h_{i}\left(s_{i}\right)$. Define $g_{i}: L \rightarrow M$ by $g_{i}(x)=r_{i} h_{i}(x)$. Then the $g_{i}$ are $R$-linear maps $L \rightarrow M$ and

$$
z=\sum_{i=1}^{k} g_{i}\left(s_{i}\right)
$$

Lemma 8.4. Let $L$ be an $R$-module and $S$ a subset of $L$. Then the submodule selector $\operatorname{tr}_{S, L}$ is functorial, i.e. a preradical, and $\operatorname{tr}_{L}$ is idempotent.

Proof. First we prove that $\operatorname{tr}_{S, L}$ is functorial. Let $f: M \rightarrow N$ be an $R$ linear map in $\mathcal{M}$ and let $z \in \operatorname{tr}_{S, L}(M)$. Then by Lemma 8.3 , there are $R$-linear maps $g_{i}: L \rightarrow M$ and elements $s_{i} \in S$ such that $z=\sum_{i} g_{i}\left(s_{i}\right)$. Then $f \circ g_{i}$ are $R$-linear maps from $L$ to $N$, and $\sum_{i}\left(f \circ g_{i}\right)\left(s_{i}\right)=f(z)$. Hence $f(z) \in \operatorname{tr}_{S, L}(N)$, as desired.

For idempotence, let $M \in \mathcal{M}$ and $z \in \operatorname{tr}_{L}(M)$. Then there is a map $g: L \rightarrow M$ and an element $q \in L$ such that $g(q)=z$. The image of $g$ must be contained in $\operatorname{tr}_{L}(M)$, so we can view $g$ as a map $L \rightarrow \operatorname{tr}_{L}(M)$. Hence $z \in \operatorname{tr}_{L}\left(\operatorname{tr}_{L}(M)\right)$.

Example 8.5. In general, $\operatorname{tr}_{S, L}$ is not idempotent. For example, take $S$ to be an ideal $I$ and $L=R$. For any $R$-module $M$, we have $\operatorname{tr}_{I, R}(M)=I M$. In particular, $\operatorname{tr}_{I, R}(R)=I$, so this submodule selector is idempotent if and only if $I=I^{2}$.

Remark 8.6. Next, we apply the limits from Section 7 to traces. In particular, given a map $g: L \rightarrow L^{\prime}$ and $X \subseteq L$, then it is easy to see that $\operatorname{tr}_{g(X), L^{\prime}} \leq \operatorname{tr}_{X, L}$. In particular, if $S \rightarrow S^{\prime}$ is a map of $R$-algebras, then $\operatorname{tr}_{S^{\prime}} \leq \operatorname{tr}_{S}$. Hence, an inverse system of algebras leads to a direct system of 
traces. In particular, if $I$ is an ideal of $R$, then we have natural surjections $R / I^{n+1} \rightarrow R / I^{n}$, and we have

$$
\left(\stackrel{\lim }{\longrightarrow} \operatorname{tr}_{R / I^{n}}\right)(M)=\bigcup_{n \in \mathbb{N}}\left(0: M I^{n}\right)=H_{I}^{0}(M) .
$$

The following condition helps us relate different traces of the same module.

Definition 8.7. Let $L$ and $M$ be $R$-modules. We say that $L$ generates $M$ if some direct sum of copies of $L$ surjects onto $M$. In particular, $L$ generates $M$ if there is a surjection $L \rightarrow M$.

The following lemma is well-known, but appears in particular in Lin17, Proposition 2.8].

Lemma 8.8. If $L \rightarrow M$ is a surjection of $R$-modules, or more generally if $L$ generates $M$, then $\operatorname{tr}_{M}(N) \leq \operatorname{tr}_{L}(N)$.

As a consequence, we get the following result concerning traces with respect to chains of increasing cyclic modules.

Lemma 8.9. Let $L$ be an $R$-module and $\left\{z_{n}\right\}_{n \in \mathbb{N}} \subseteq L$. If

$$
R z_{1} \subseteq R z_{2} \subseteq \ldots \subseteq R z_{n} \subseteq \ldots,
$$

then $\operatorname{tr}_{R z_{j}} \leq \operatorname{tr}_{R z_{j+1}}$ for all $j \in \mathbb{N}$.

Proof. For all $j \in \mathbb{N}, \operatorname{ann}_{R}\left(z_{j}\right) \supseteq \operatorname{ann}_{R}\left(z_{j+1}\right)$. Hence we have surjections

$$
R / \operatorname{ann}_{R}\left(z_{j+1}\right) \rightarrow R / \operatorname{ann}_{R}\left(z_{j}\right) .
$$

By Remark $8.2, \operatorname{tr}_{R_{z_{j}}}=\operatorname{tr}_{R / \operatorname{ann}_{R}\left(z_{j}\right)}$, and hence the result follows.

The next result was mentioned at the end of Section 7

Proposition 8.10. Let $\Gamma$ be a directed poset and $\left\{L_{j}\right\}_{j \in \Gamma}$ a set of $R$-modules such that $\operatorname{tr}_{L_{i}} \geq \operatorname{tr}_{L_{j}}$ for all $i \leq j$. In particular, it may be the case that $L_{i}$ generates $L_{j}$ whenever $i \leq j$, or $\left\{L_{j}\right\}$ may be a set of $R$-algebras, with $R$ algebra maps $L_{i} \rightarrow L_{j}$ whenever $i \leq j$. Let $L=\lim _{\longrightarrow} L_{j}$. Then $\operatorname{tr}_{L} \leq \lim _{j} \operatorname{tr}_{L_{j}}$.

Proof. By the hypothesis, for $i \leq j, \operatorname{tr}_{L_{i}} \geq \operatorname{tr}_{L_{j}}$. Hence $\operatorname{tr}_{L} \leq \operatorname{tr}_{L_{j}}$ for all $j \in \Gamma$. This implies that $\operatorname{tr}_{L} \leq \bigcap_{j} \operatorname{tr}_{L_{j}}$, which by definition is $\lim _{j} \operatorname{tr}_{L_{j}}$.

We end this subsection by noting the following, which amounts to a general principle regarding how to deal with traces when working with modules over various rings.

Proposition 8.11. Let $T$ be an R-algebra, $L$ a $T$-module, and $X \subseteq L$ a subset. Let $U$ be the $T$-submodule of $L$ generated by $X$. Then $\operatorname{tr}_{X, L}=\operatorname{tr}_{U, L}$, where in both cases, tr means $\operatorname{tr}^{(R)}$.

Proof. The fact that $\operatorname{tr}_{X, L} \leq \operatorname{tr}_{U, L}$ follows by definition and from the fact that $X$ is a subset of $U$.

Conversely, let $M$ be an $R$-module. To show that $\operatorname{tr}_{U, L}(M) \subseteq \operatorname{tr}_{X, L}(M)$, it suffices to show that for any $R$-linear $g: L \rightarrow M$ and any $y \in U$, we have 
$g(y) \in \operatorname{tr}_{X, L}(M)$. So take such $y$ and $g$. Since $U$ is generated by $X$ as a $T$-module, there exist $n \in \mathbb{N}, t_{i} \in T$, and $x_{i} \in X$ such that $y=\sum_{i=1}^{n} t_{i} x_{i}$.

For each $1 \leq i \leq n$, consider the $R$-linear map $h_{i}: L \rightarrow M$ given by $h_{i}(\ell):=g\left(t_{i} \ell\right)$. This is $R$-linear because for any $\ell, \ell^{\prime} \in L$ and $r \in R$, we have

$$
h_{i}\left(\ell+r \ell^{\prime}\right)=g\left(t_{i}\left(\ell+r \ell^{\prime}\right)\right)=g\left(t_{i} \ell\right)+r g\left(t_{i} \ell^{\prime}\right)=h_{i}(\ell)+r h_{i}\left(\ell^{\prime}\right) .
$$

Then $g(y)=\sum_{i=1}^{n} g\left(t_{i} x_{i}\right)=\sum_{i=1}^{n} h_{i}\left(x_{i}\right) \in \operatorname{tr}_{X, L}(M)$.

Trace behaves well under flat base change. The next result generalizes a result of Lindo [Lin17, Proposition 2.8(viii)] to the setting of $(X, L)$-trace, using a similar proof technique.

Theorem 8.12. Let $B$ be an $R$-module, $X$ a subset of $B$, and $S$ a commutative $R$-algebra. Then for any $R$-module $M$,

$$
\operatorname{im}\left(\operatorname{tr}_{X, B}(M) \otimes_{R} S \rightarrow M \otimes_{R} S\right) \subseteq \operatorname{tr}_{X^{\prime}, B \otimes_{R} S}\left(M \otimes_{R} S\right)
$$

as $S$-submodules of $M \otimes_{R} S$, where $X^{\prime}=\{x \otimes s \mid x \in X, s \in S\} \subseteq B \otimes_{R} S$. If $B$ is finitely presented and $S$ is flat over $R$, then we have equality.

Proof. Let $z \in \operatorname{tr}_{X, B}(M)$ and $s \in S$. Then there exist $n \in \mathbb{N}$ and $R$-linear maps $f_{i}: B \rightarrow M$ and elements $x_{i} \in X$ for $1 \leq i \leq n$ such that $z=\sum_{i=1}^{n} f_{i}\left(x_{i}\right)$. Then the maps $f_{i} \otimes 1: B \otimes_{R} S \rightarrow M \otimes_{R} S$ are $S$-linear, and $z \otimes s=\sum_{i=1}^{n}\left(f_{i} \otimes 1\right)\left(x_{i} \otimes s\right)$. Hence, $\operatorname{im}\left(\operatorname{tr}_{X, B}(M) \otimes_{R} S \rightarrow M \otimes_{R} S\right) \subseteq \operatorname{tr}_{X^{\prime}, B \otimes_{R} S}\left(M \otimes_{R} S\right)$.

Now suppose $S$ is a flat $R$-algebra and $B$ a finitely generated $R$-module. Let $c \in \operatorname{tr}_{X^{\prime}, B \otimes_{R} S}\left(M \otimes_{R} S\right)$. Then there are $S$-linear maps $g_{i}: B \otimes_{R} S \rightarrow$ $M \otimes_{R} S$ and elements $d_{i} \in X^{\prime}$ such that $c=\sum_{i} g_{i}\left(d_{i}\right)$. We have $d_{i}=\sum_{k} x_{i k} \otimes s_{i k}$ for elements $x_{i k} \in X$ and $s_{i k} \in S$. Moreover, by the assumptions on $B$ and $S$, the following map is an isomorphism (see e.g. [EJ00, Lemma 3.2.4]):

$$
\varphi: \operatorname{Hom}_{R}(B, M) \otimes S \rightarrow \operatorname{Hom}_{S}\left(B \otimes_{R} S, M \otimes_{R} S\right)
$$

sending $f \otimes s \mapsto(b \otimes t \mapsto f(b) \otimes s t)$. For each $i$,

$$
g_{i}=\varphi\left(\sum_{j} f_{i j} \otimes t_{i j}\right)
$$

for $R$-linear maps $f_{i j}: B \rightarrow M$ and elements $t_{i j} \in S$. We have

$$
\begin{aligned}
g_{i}\left(d_{i}\right) & =\varphi\left(\sum_{j} f_{i j} \otimes t_{i j}\right)\left(\sum_{k} x_{i k} \otimes s_{i k}\right) \\
& =\sum_{j, k} \varphi\left(f_{i j} \otimes t_{i j}\right)\left(x_{i k} \otimes s_{i k}\right) \\
& =\sum_{j, k}\left(f_{i j}\left(x_{i k}\right) \otimes t_{i j} s_{i k}\right) \in \operatorname{tr}_{X, B}(M) \otimes_{R} S .
\end{aligned}
$$

It follows that

$$
c=\sum_{i} g_{i}\left(d_{i}\right) \in \operatorname{tr}_{X, B}(M) \otimes_{R} S
$$


We include a statement of Lindo's result, which deals with the special case corresponding to the usual trace operation, for the sake of keeping the paper self-contained:

Corollary 8.13 ([Lin17, Proposition 2.8(viii)]). If $B$ is a finitely presented $R$-module and $S$ is a flat commutative R-algebra, then $\operatorname{tr}_{B}(R) \otimes_{R} S=\operatorname{tr}_{B \otimes_{R} S}(S)$. In particular, for any prime ideal $\mathfrak{p} \in \operatorname{Spec} R$, we have $\operatorname{tr}_{B}(R)_{\mathfrak{p}}=\operatorname{tr}_{B_{\mathfrak{p}}}\left(R_{\mathfrak{p}}\right)$ and $\left.\operatorname{tr}_{B}(R) \widehat{R_{\mathfrak{p}}}=\operatorname{tr}_{B_{\mathfrak{p}}\left(R_{\mathfrak{p}}\right.}\right)$.

Example 8.14. We give an example where $B$ is not finitely generated and the formation of the trace ideal does not commute with localization, even though the ring is complete. Let $R$ be a complete DVR, e.g. $k[[t]]$, let $\mathfrak{m}=(t)$ be its maximal ideal, let $B$ be its fraction field, and let $P:=(0) R$. Then $\operatorname{tr}_{B}(R)=0$. To see this, let $g: B \rightarrow R$ be $R$-linear and $\alpha=g(1)$. Then for any positive integer $n$, we have $\alpha=g(1)=g\left(t^{n} \cdot 1 / t^{n}\right)=t^{n} g\left(1 / t^{n}\right) \in \mathfrak{m}^{n}$. Thus, $\alpha \in \bigcap_{n} \mathfrak{m}^{n}=0$, whence $g=0$. On the other hand, since $B_{P}=R_{P}$, $\operatorname{tr}_{B_{P}}\left(R_{P}\right)=R_{P}$.

Dual of trace is module-torsion. We continue the convention that $R$ is a commutative ring with identity.

Definition 8.15. Let $L$ be an $R$-module, and $S \subseteq L$. Then we define the module torsion with respect to $S \subseteq L$ to be the submodule selector given by

$$
\operatorname{tom}_{S, L}(M):=\left\{z \in M \mid \forall s \in S, s \otimes z=0 \text { in } L \otimes_{R} M\right\} .
$$

If $S=\{x\}$ is a singleton, we write $\operatorname{tom}_{x, L}:=\operatorname{tom}_{\{x\}, L}$. If $S=L$, we write $\operatorname{tom}_{L}:=\operatorname{tom}_{L, L}$.

Remark 8.16. We note the following easy facts, parallel to Remark 8.2

(1) For any $R$-module $L$ and any set $S \subseteq L, \operatorname{tom}_{S, L}$ is a submodule selector on $\mathcal{M}$. In particular, $\operatorname{tom}_{x, L}$ and tom $_{L}$ are submodule selectors.

(2) When $L=A$ is an $R$-algebra, $\operatorname{tom}_{A}=\operatorname{tom}_{1, A}$.

(3) When $I$ is an ideal of $R$ and $L, N$ are $R$-modules, $\operatorname{tom}_{L / I L}(N)=$ $\pi^{-1}\left(\operatorname{tom}_{L}(N / I N)\right)$, where $\pi: N \rightarrow N / I N$ is the natural surjection. In particular, $\operatorname{tom}_{R / I}(N)=I N$.

(4) When $I$ is an ideal of $R, \operatorname{tom}_{I, R}(N)=\left(0:_{N} I\right)$.

(5) If $L$ is an $R$-module and $x \in L$, then $\operatorname{tom}_{R x} \leq \operatorname{tom}_{x, L}$ : consider the composition $M \rightarrow R x \otimes_{R} M \rightarrow L \otimes_{R} M$, where the first map sends $m \mapsto x \otimes_{R} m$.

(6) However, the converse is false. Let $x$ be a regular element in $R$ and let $M=R /(x)$. Then $\operatorname{tom}_{R x}(M)=\operatorname{tom}_{R / \operatorname{ann}_{R}(x)}(M)=\operatorname{ann}_{R}(x) M=0$ but $\operatorname{tom}_{x, R}(M)=\operatorname{ann}_{M}(x)=M$.

Next we give a kind of dual to Proposition 8.11

Proposition 8.17. Let $T$ be an R-algebra, $L$ a $T$-module, and $X \subseteq L a$ subset. Let $U$ be the $T$-submodule of $L$ generated by $X$. Then tom ${ }_{X, L}=$ tom $_{U, L}$, where in both cases, tom means tom ${ }^{(R)}$. 
Proof. The fact that $\operatorname{tom}_{U, L} \leq \operatorname{tom}_{X, L}$ follows by definition and from the fact that $X$ is a subset of $U$.

For the opposite direction, let $M$ be an $R$-module and $z \in \operatorname{tom}_{X, L}(M)$. Let $u \in U$. Then there exist $n \in \mathbb{N}, t_{i} \in T$, and $x_{i} \in X$ such that $u=\sum_{i=1}^{n} t_{i} x_{i}$. Using the left $T$-module structure of $L \otimes_{R} M$, we have in that module

$$
u \otimes z=\left(\sum_{i=1}^{n} t_{i} x_{i}\right) \otimes z=\sum_{i=1}^{n} t_{i} \cdot\left(x_{i} \otimes z\right)=\sum_{i=1}^{n} t_{i} \cdot 0=0 .
$$

Theorem 8.18. Let $R$ be a complete local Noetherian ring, $L$ an $R$-module,

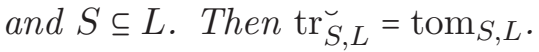

Proof. Let $M$ be a Matlis-dualizable module. We first let $z \in \operatorname{tom}_{S, L}(M)$. That is, $z \in M$ such that $s \otimes z=0$ in $L \otimes_{R} M$ for all $s \in S$. By Theorem 3.3, we want to show that $g(z)=0$ for all $g \in \operatorname{tr}_{S, L}\left(M^{\vee}\right)$. So let $g \in \operatorname{tr}_{S, L}\left(M^{\vee}\right)$. Then there exist $R$-linear maps $\varphi_{i}: L \rightarrow M^{\vee}$ and $s_{i} \in S$ such that $g=\sum_{i=1}^{n} \varphi_{i}\left(s_{i}\right)$. Let $\psi_{i}: L \otimes_{R} M \rightarrow E$ be the maps corresponding to the $\varphi_{i}$ by Hom-tensor adjointness. Since $s_{i} \otimes z=0$ in $L \otimes M$, we have

$$
g(z)=\sum_{i=1}^{n} \varphi_{i}\left(s_{i}\right)(z)=\sum_{i=1}^{n} \psi_{i}\left(s_{i} \otimes z\right)=\sum_{i=1}^{n} \psi_{i}(0)=0 .
$$

This implies that $\operatorname{tom}_{S, L} \subseteq\left(\operatorname{tr}_{S, L}\right)^{\smile}$.

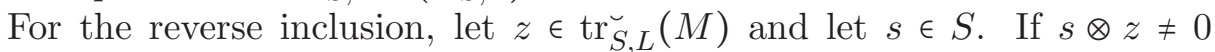
in $L \otimes_{R} M$, then since $\operatorname{Hom}_{R}(-, E)$ never kills a nonzero module, we have $(R \cdot(s \otimes z))^{\vee} \neq 0$. Since $E$ is injective, it follows that there is an $R$-linear map $\varphi: L \otimes_{R} M \rightarrow E$ such that $\varphi(s \otimes z) \neq 0$.

Let $\psi: L \rightarrow M^{\vee}$ be the corresponding map that arises from Hom-tensor adjointness. Then we have

$$
\psi(s)(z)=\varphi(s \otimes z) \neq 0 .
$$

On the other hand, since $z \in \operatorname{tr}_{S, L}^{\sim}(M)$ and $\psi(s) \in \operatorname{tr}_{S, L}\left(M^{\vee}\right)$, Theorem 3.3 yields that $z \in \operatorname{ker} \psi(s)$, contradicting the display above. Hence, $s \otimes z=0$ in $L \otimes_{R} M$ for all $s \in S$, whence $z \in \operatorname{tom}_{S, L}(M)$, as was to be shown.

Note that the $R$-module $L$ has no restriction on it-it does not have to be either finitely generated or artinian.

Corollary 8.19. Let $R$ be a complete local ring, $L$ an $R$-module, and $S \subseteq L$. Then $\operatorname{tom}_{S, L}$ is functorial and $\operatorname{tom}_{L}$ is co-idempotent.

Proof. By Theorem 8.18, tom ${ }_{S, L}$ is dual to $\operatorname{tr}_{S, L}$. By Lemma 8.4, $\operatorname{tr}_{S, L}$ is functorial and $\operatorname{tr}_{L}$ is idempotent, and so by Theorems 8.18 and 3.2. $\operatorname{tom}_{S, L}$ is functorial and $\operatorname{tom}_{L}$ is co-idempotent.

Remark 8.20. Recall R.G16, Definition 2.3] that for an $R$-module $L$, the module closure given by $L$ is given by the formula

$$
N_{M}^{\mathrm{cl}_{L}}:=\{u \in M \mid \forall x \in L, x \otimes u \in \operatorname{im}(L \otimes N \rightarrow L \otimes M)\},
$$


whenever $M$ is an $R$-module and $N$ a submodule of $M$. In the complete local Noetherian case, it follows from Theorem 8.18 that in the notation of Section 4, we have $\sigma\left(\mathrm{cl}_{L}\right)=\operatorname{tom}_{L}$, and hence by the above theorem we have

$$
i\left(\mathrm{cl}_{L}\right)=\operatorname{tr}_{L} \text {. }
$$

That is, the L-trace is the interior operation dual to the module closure defined by $L$. This demonstrates how our framework can be used to achieve results comparable to those in [PR20].

Corollary 8.21. If $R$ is a complete local Noetherian ring and $Q$ is an $R$ -

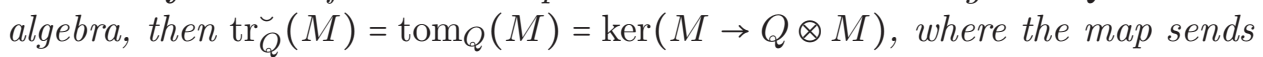
$z \mapsto 1 \otimes z$.

Corollary 8.22. Let $I$ be an ideal of a complete local Noetherian ring $R$.

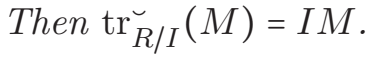

The following result connects tom to the 0th local cohomology module, similar to Remark 8.6.

Corollary 8.23. Let $I$ be an ideal of a complete local Noetherian ring $R$. Then for any $R$-module $M$,

$$
\begin{aligned}
& \left(H_{I}^{0}\right)^{\smile}(M)=\left(\stackrel{\lim }{\longrightarrow} \operatorname{tr}_{R / I^{n}}\right)^{\smile}(M)=\left(\lim _{\longleftarrow}\left(\operatorname{tr}_{R / I^{n}}^{\smile}\right)\right)(M) \\
& =\left(\lim _{\longleftarrow}\left(\operatorname{tom}_{R / I^{n}}\right)\right)(M)=\bigcap_{n}\left(I^{n} M\right)=\operatorname{ker}\left(M \rightarrow \widehat{M}^{I}\right) .
\end{aligned}
$$

The next example demonstrates that direct limits do not commute with taking tom.

Example 8.24. Let $(R, m)$ be a complete DVR (for example $\widehat{\mathbb{Z}_{(2)}}$ or $k[[x]]$ ), $\pi \in R$ such that $m=(\pi), N_{j}=\frac{1}{\pi^{j}} R$, and $K=\operatorname{Frac}(R)$. Then $K=\underline{\lim _{\longrightarrow}} N_{j}$. Since $N_{j} \cong R$ for all $j, \operatorname{tr}_{N_{j}}(R)=R$. However, $\operatorname{tr}_{K}(R)=0$. So

$$
\left(\lim _{j} \operatorname{tr}_{N_{j}}\right)(R)=\bigcap_{j}\left(\operatorname{tr}_{N_{j}}(R)\right)=\bigcap_{j} R=R,
$$

but

$$
\operatorname{tr}_{\left(\lim _{\rightarrow j} N_{j}\right)}(R)=\operatorname{tr}_{K}(R)=0 .
$$

Hence $\operatorname{tom}_{K} \ngtr \lim _{\longrightarrow} \operatorname{tom}_{N_{j}}$.

Proposition 8.25. Let $(R, \mathfrak{m})$ be a complete local Noetherian ring and $Q$ an $R$-module such that the quotient module $Q / J Q$ is $\mathfrak{m}$-adically separated for any ideal $J$ of $R$. Then the last condition of Theorem 5.5 applies to tom $_{Q}$, and hence to $\mathrm{cl}_{Q}$. Thus,

$$
\bigcap_{\lambda(R / I)<\infty} \operatorname{ann}\left(\operatorname{tom}_{Q}(R / I)\right)=\bigcap_{I \text { ideal of } R}\left(I: I^{\mathrm{cl}_{Q}}\right) .
$$

In particular, this holds when $Q$ is finitely generated. 
Proof. Let $J$ be an ideal of $R$. Then $J=\bigcap_{n}\left(J+\mathfrak{m}^{n}\right)$ by the Krull intersection theorem, and if $a \in \bigcap_{n}\left(J+\mathfrak{m}^{n}\right)^{\mathrm{cl}_{Q}}$, then for any $q \in Q$, we have $a q \in \bigcap_{n}(J+$ $\left.\mathfrak{m}^{n}\right) Q=J Q$ by assumption of $\mathfrak{m}$-adic separatedness of $Q / J Q$. Hence $a \in J^{\mathrm{cl}_{Q}}$, whence $J^{\mathrm{cl}_{Q}}=\bigcap_{n}\left(J+\mathfrak{m}^{n}\right)^{\mathrm{cl}_{Q}}$ as required.

\section{Special CASES: TORSION AND Divisibility FOR A MUltiplicAtive} SET

As another application of our setup, we consider the notions of torsion and divisibility with respect to a multiplicative set. These are known in a general way to be dual to each other; we clarify the nature of the duality in the context of Matlis duality over a complete local ring.

In this section, $R$ is a commutative ring with identity.

Accordingly, recall the following definitions as given in [EY12, §4]:

Definition 9.1. Let $R$ be a ring, $W \subseteq R$ a multiplicatively closed set, and $M$ an $R$-module. We say $M$ is

- $W$-torsion free if for all $w \in W$, the homothety map on $M$ induced by $w$ is injective, or equivalently, if the natural map $M \rightarrow W^{-1} R \otimes_{R} M$ given by $z \mapsto \frac{z}{1}$ is injective.

- $W$-divisible if for all $z \in M$ and all $w \in W$, there is some $y \in M$ such that $z=w y$, or equivalently if for all $w \in W$, the homothety map on $M$ induced by $w$ is surjective.

- $h_{W}$-divisible if there is a free $W^{-1} R$-module $N$ and a surjective $R$ linear map $N \rightarrow M$, or if equivalently (see [EY12, Lemma 4.1]) the evaluation map $\operatorname{Hom}_{R}\left(W^{-1} R, M\right) \rightarrow M$ given by $f \mapsto f(1 / 1)$ is surjective.

When used without modifier, typically $R$ is an integral domain and $W=$ $R \backslash\{0\}$. Divisibility (and h-divisibility) are weak forms of injectivity, whereas torsion-freeness is a weak form of flatness, and in general are very important properties of modules. Hence it behooves us to consider these notions in submodule selector terms.

Definition 9.2. Let $W \subseteq R$ be a multiplicatively closed set, and $\mathcal{M}$ a class of modules closed under taking submodules and quotient modules.

- We define the $W$-torsion submodule selector to $W$ by setting to $W(M):=$ the kernel of the localization map $M \rightarrow W^{-1} M$, or equivalently, the set of $z \in M$ such that there exists $w \in W$ such that $w z=0$. Since $W^{-1} M \cong W^{-1} R \otimes_{R} M$, with the localization map corresponding to the map $z \mapsto 1 \otimes z$, it follows that to $W=\operatorname{tom}_{W^{-1} R}$.

- We define the $W$-divisible submodule selector $\operatorname{div}_{W}$ by $\operatorname{setting}_{\operatorname{div}_{W}}(M)$ := the sum (hence the union) of the $W$-divisible submodules of $M$.

Proposition 9.3. Let $W$ be a multiplicatively closed set.

(1) An R-module $M$ is $W$-torsion free if and only if $\operatorname{to}_{W}(M)=0$.

(2) An R-module $M$ is $W$-divisible if and only if $\operatorname{div}_{W}(M)=M$. 
(3) An R-module $M$ is $h_{W}$-divisible if and only if $\operatorname{tr}_{W^{-1} R}(M)=M$.

(4) For any $R$-module $M, \operatorname{tr}_{W^{-1} R}(M) \subseteq \operatorname{div}_{W}(M)$. That is,

$$
\operatorname{tr}_{W^{-1} R} \leq \operatorname{div}_{W} .
$$

(5) to $_{W}=\underset{w \in W}{\lim _{w}} \operatorname{tr}_{R /(w)}$.

(6) $\operatorname{div}_{W}$ is idempotent and functorial.

(7) tow is idempotent, functorial, and co-idempotent.

(8) If $R$ is a complete local Noetherian ring and $M$ is Matlis-dualizable, then $\operatorname{div}_{W}(M)=\operatorname{tr}_{W^{-1} R}(M)$.

(9) If $R$ is a complete local Noetherian ring, then when applied to Matlisdualizable modules, we have to $W=\operatorname{div}_{W}$.

(10) In this case, $\operatorname{div}_{W}(M)=\bigcap_{w \in W} w M$.

Proof. Part (1) is by definition.

For part (2), the "only if" direction is clear. Conversely, $\operatorname{suppose} \operatorname{div}_{W}(M)=$ $M$. Let $z \in M$ and $w \in W$. Then there is some $W$-divisible submodule $L$ of $M$ with $z \in L$. Hence $z \in w L \subseteq w M$, whence the homothety is surjective.

Part (3) follows from the definition and Remark 8.2(2).

For part (4), let $z \in \operatorname{tr}_{W^{-1} R}(M)$ and $w \in W$. Then there is some $R$ linear map $g: W^{-1} R \rightarrow M$ with $z=g(1 / 1)$. Thus, $z=g(1 / 1)=g(w / w)=$ $w g(1 / w) \in w M$.

For part (5), first note that modules of the form $R /(w), w \in W$ form an inverse limit system, since if $w, w^{\prime} \in W$, then also $w w^{\prime} \in W$, and we have $R /\left(w w^{\prime}\right) \rightarrow R /(w)$ and $R /\left(w w^{\prime}\right) \rightarrow R /\left(w^{\prime}\right)$ via the canonical maps. Hence as in 8 , one can discuss a direct limit of traces. In particular, for any $R$-module $M$, we have

$$
\begin{aligned}
\left(\underset{w \in W}{\lim _{\vec{W}}} \operatorname{tr}_{R /(w)}\right)(M) & =\bigcup_{w \in W} \operatorname{tr}_{R /(w)}(M) \\
& =\bigcup_{w \in W}\left(0:_{M} w\right)=\operatorname{to}_{W}(M) .
\end{aligned}
$$

To see part (6), let $z \in \operatorname{div}_{W}(M)$. Then there is some $W$-divisible submodule $L$ of $M$ such that $z \in L$. But since $L \subseteq \operatorname{div}_{W}(M)$, it follows that $z \in \operatorname{div}_{W}\left(\operatorname{div}_{W}(M)\right)$. For functoriality, first note that if $g: L \rightarrow M$ is an $R$-linear map and $L^{\prime}$ a $W$-divisible submodule of $L$, then $g\left(L^{\prime}\right)$ is $W$ divisible. For if $u=g(t) \in g\left(L^{\prime}\right)$ and $w \in W$, then since $t \in L^{\prime}$, there is some $s \in L^{\prime}$ with $t=w s$, whence $u=g(t)=g(w s)=w g(s) \in w g\left(L^{\prime}\right)$. Now let $z \in \operatorname{div}_{W}(L)$. Then there is a $W$-divisible submodule $L^{\prime}$ of $L$ with $z \in L^{\prime}$. But then $g(z) \in g\left(L^{\prime}\right)$, which is a $W$-divisible submodule of $M$, whence $g(z) \in \operatorname{div}_{W}(M)$.

To see part (7), let $z \in \operatorname{to}_{W}(M)$. Then there is some $w \in W$ with $w z=0$, and $z \in \operatorname{to}_{W}(M)$; hence $z \in \operatorname{to}_{W}\left(\operatorname{to}_{W}(M)\right)$. For functoriality, let $g: L \rightarrow$ $M$ be a map in $\mathcal{M}$ and $z \in \operatorname{to}_{W}(L)$. Then $w z=0$ for some $w \in W$, so $w g(z)=g(w z)=g(0)=0$, whence $g(z) \in \operatorname{to}_{W}(M)$. For co-idempotence, let $N=M / \operatorname{to}_{W}(M)$, and $z \in M$ such that $\bar{z} \in \operatorname{to}_{W}(N)$. Then for some $w \in W$, 
$w \bar{z}=\overline{w z}=\overline{0}$, whence $w z \in \operatorname{to}_{W}(M)$. Then there is some $v \in W$ with $v w z=0$, so that $z \in \operatorname{to}_{W}(M)$, whence $\bar{z}=\overline{0}$. Hence $\operatorname{to}_{W}\left(M / \operatorname{to}_{W}(M)\right)=0$.

For part (8), first recall [EY12, Lemma 4.6] that in this context, a module is divisible if and only if it is $\mathrm{h}_{W}$-divisible. By part (4), we need only show that $\operatorname{div}_{W}(M) \subseteq \operatorname{tr}_{W^{-1} R}(M)$. Let $z \in \operatorname{div}_{W}(M)$. Since $\operatorname{div}_{W}(M)$ is $W$-divisible (by (2) and (6)) and hence $\mathrm{h}_{W}$-divisible (by the result quoted above), there is some $R$-linear map $g: W^{-1} R \rightarrow \operatorname{div}_{W}(M)$ with $z=g(1)$. Let $j: \operatorname{div}_{W}(M) \hookrightarrow M$ be the inclusion map. Then $f:=j \circ g: W^{-1} R \rightarrow M$ is $R$-linear with $z=f(1)$, so $z \in \operatorname{tr}_{W^{-1} R}(M)$.

For part (9), we need only quote part (8) and Corollary 8.21 .

Finally, part (10) follows from part (5), Corollary 8.22, and Proposition 7.7.

Remark 9.4. It follows that when restricted to Matlis-dualizable modules over complete local Noetherian rings, $\operatorname{div}_{W}$ and to $W$ are co-idempotent preradicals. Thus they have associated residual closure operations. Namely,

$$
L_{M}^{\mathrm{to}_{W}}=\pi^{-1}\left(\operatorname{to}_{W}(M / L)\right)=\bigcup_{w \in W}\left(L:_{M} w\right)
$$

and

$$
L_{M}^{\operatorname{div}_{W}}=\pi^{-1}\left(\operatorname{div}_{W}(M / L)\right)=\bigcap_{w \in W}(L+w M),
$$

where $\pi: M \rightarrow M / L$ is the canonical surjection.

\section{Special Case: tight Closure and integral Closure}

Tight closure inspired much of the study of residual closure operations in commutative algebra. We apply our framework to tight closure and note that it is dual to tight interior as defined in ES14.

In this section, let $R$ be a commutative Noetherian ring with identity.

Definition 10.1. Let $R$ be a commutative Noetherian ring of prime characteristic $p>0, F^{e}$ denote the eth Frobenius functor, and $N \subseteq M$ be $R$ modules. Let ${ }^{e} M$ denote the set of elements of $M$ with the same additive structure as $M$ and the $R-R$ bimodule structure given by $r \cdot\left({ }^{e} x\right) \cdot s={ }^{e}\left(r^{q} s x\right)$ for $r, s \in R$ and ${ }^{e} x \in{ }^{e} M$. We define $N_{M}^{[q]}$ to be the kernel of the map $F^{e}(M) \rightarrow F^{e}(M / N)$ and $u^{q}:=1 \otimes_{R} u \in{ }^{e} R \otimes_{R} M$. The tight closure of $N$ in $M$ is the set of $u \in M$ such that there exists a $c \in R^{\circ}$ with

$$
c u^{q} \in N_{M}^{[q]}
$$

for all $q=p^{e} \gg 0$ [HH90, Section 8].

Assume further that $R_{\text {red }}$ is $F$-finite. For $q_{0}$ a power of $p$ and $c \in R^{\circ}$, let

$$
M_{*}\left[c, q_{0}\right]:=\sum_{q \geq q_{0}} \operatorname{im}\left(\operatorname{Hom}_{R}\left({ }^{e}(R), M\right) \rightarrow M\right),
$$

where the map sends $g:{ }^{e} R \rightarrow M$ to $g\left({ }^{e} c\right)$. The tight interior of $M$, denoted $M_{\star}$, is

$$
M_{*}:=\bigcap_{c \in R^{\circ}} \bigcap_{q_{0} \geq 1} M_{*}\left[c, q_{0}\right]
$$


ES14.

Proposition 10.2. Let $R$ be a complete local Noetherian ring of characteristic $p>0$ and let $\alpha(M)=0_{M}^{*}$, where * denotes tight closure.

(1) $\alpha$ is functorial and co-idempotent.

(2) If $R$ is complete local and reduced, then viewing $\alpha$ as a submodule selector on finitely generated and Artinian R-modules, $\alpha^{\sim}$ is tight interior.

(3) Tight interior is functorial and idempotent.

Proof. (1) [HH90, Section 8] tells us that tight closure is functorial and idempotent. The result then follows from Proposition 2.6.

(2) [ES14, Corollary 4.6].

(3) These are in [ES14]: functorial is Lemma 2.1, idempotent is Proposition 2.7, and localization is Corollary 2.11.

We recover the following well-known result about tight closure from our framework.

Proposition 10.3. Let $(R, m)$ be a complete local Noetherian ring of characteristic $p>0$. Then tight closure satisfies the hypotheses of Theorem 5.5, and hence its conclusions.

Proof. This follows from Remark 5.6] and Theorem 5.5.

Another commonly used closure operation is integral closure (see, for example [HS06]). We use here the residual version of integral closure, defined in [EU]:

Definition 10.4 ([EU]). Let $L \subseteq F$ be $R$-modules, where $F$ is free. Let $S$ be the symmetric algebra over $R$ defined by $F$, with the natural grading, and let $T$ be the subring of $S$ induced by the inclusion $L \subseteq F$. Note that $S$ is $\mathbb{N}$-graded and generated in degree 1 over $R$, and $T$ is an $\mathbb{N}$-graded subring of $S$, also generated over $R$ in degree 1 . The integral closure of $L$ in $F$, denoted $L_{F}^{-}$is the degree 1 part of the integral closure of the subring $T$ of $S$.

Now let $L \subseteq M$ be $R$-modules and $\pi: F \rightarrow M$ a surjection of a free module $F$ onto $M$. Let $K=\pi^{-1}(L)$. The liftable integral closure of $L$ in $M$ is

$$
L_{M}^{\vdash}:=\pi\left(K_{F}^{\vdash}\right) \text {. }
$$

Remark 10.5. In [EU, Proposition 2.2], Epstein and Ulrich show that this definition is independent of the choice of free module $F$ and surjection $\pi: F \rightarrow M$.

Proposition 10.6. Liftable integral closure is residual, idempotent, surjectionfunctorial, and order-preserving on ambient modules and on submodules. Hence setting $\alpha(M)=0_{M}^{\leftarrow}, \alpha$ is functorial and co-idempotent. 
Proof. The first line follows from [EU, Proposition 2.4], and the second from Proposition 2.6.

Proposition 10.7. Let $R$ be a complete local Noetherian ring. Setting $\alpha(M)=0_{M}^{\leftarrow}, \alpha$ satisfies the hypotheses of Theorem [5.5, and hence its conclusions. In particular,

$$
\alpha^{\smile}(R)=\operatorname{ann}(\alpha(E))=\operatorname{ann}\left(0_{E}^{\llcorner}\right)
$$

and

$$
\widetilde{\alpha_{f}}(R)=\operatorname{ann}\left(\alpha_{f}(E)\right)=\bigcap_{\text {an ideal }}\left(I: I^{-}\right) .
$$

This implies that $\alpha^{\smile}(R)$ agrees with $\tau_{\mathcal{M}}$ as defined in $[\mathrm{EU}]$, and $\alpha_{f}^{\smile}(R)$ agrees with $\tau_{\mathcal{I}}$.

Proof. This follows from Remark [5.6, Theorem [5.5, and Definition/Proposition 3.1 of $[\mathrm{EU}]$.

As a consequence, we know the following about $\alpha^{\complement}(R)$ :

Corollary 10.8 ([EU]). Let $(R, m)$ be a complete local Noetherian ring.

(1) If $\operatorname{dim}(R)=0$, then $\alpha(M)=m M$ and $\alpha^{\smile}(R)=\operatorname{Soc}(R)$ EU, Propositions 4.1 and 4.2$]$.

(2) If $\operatorname{dim}(R)=1$ and $R$ has an infinite residue field, then $R$ is CohenMacaulay if and only if $\widetilde{\alpha_{f}}(R)$ is equal to the conductor of $R$ in its integral closure. Otherwise the conductor is equal to $R$ whereas

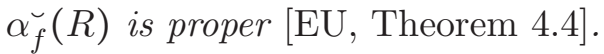

(3) If $R$ is either excellent or the homomorphic image of a Gorenstein local ring, $\operatorname{dim}(R) \geq 2$, and $R$ is equidimensional with no embedded

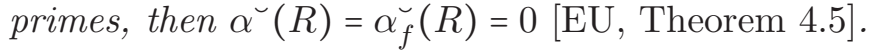

\section{Special Case: almost Ring theory, Heitmann Closures and POST-2016 MIXED CHARACTERISTIC CLOSURES}

Next we apply our structure to the example of a mixed characteristic closure operation defined in PR20]. This demonstrates how our results can be applied to the almost ring theory setting, and to a closure operation that appears distinct from a module closure.

Definition 11.1. Let $(R, m)$ be a complete local Noetherian ring of dimension $d>0$ and mixed characteristic $(0, p), T$ an $R$-algebra, and $\pi \in T$ such that $T$ contains a compatible system of $p$-power roots of $\pi$, i.e. a set of elements $\left\{\pi^{1 / p^{n}}\right\}_{n \geq 1}$ such that $\left(\pi^{1 / p^{n}}\right)^{p^{n}}=\pi$ and for $m<n,\left(\pi^{1 / p^{n}}\right)^{p^{m}}=\pi^{1 / p^{n-m}}$. We can denote this system of $p$-power roots of $\pi$ by $\pi^{1 / p^{\infty}}$.

Definition 11.2 (c.f. PR20]). Let $R, T$, and $\pi$ be as above. We define a closure operation $\mathrm{cl}$ by $u \in N_{M}^{\mathrm{cl}}$ if for all $n>0$,

$$
\pi^{1 / p^{n}} \otimes u \in \operatorname{im}(T \otimes N \rightarrow T \otimes M) .
$$

We describe the dual of this closure operation below. 
Proposition 11.3. Let $\alpha(M)=0_{M}^{\mathrm{cl}}$ with cl the closure operation from Definition 11.2. Under the hypotheses of Definition 11.1, let

$$
\beta(M)=\left\{z \in M: \pi^{1 / p^{n}} \otimes z=0 \in T \otimes_{R} M \text { for all } n>0\right\} .
$$

Then

$$
\beta=\alpha^{\leftrightharpoons} .
$$

Proof. By Proposition 8.11 we have $\alpha=\sum_{n>0} \operatorname{tr}_{\pi^{1 / p^{n}}, T}$. Then $\rho=\lim _{\longrightarrow n} \operatorname{tr}_{\pi^{1 / p^{n}}, T}$. By Proposition 7.7

$$
\rho^{\smile}=\lim _{n}\left(\operatorname{tr}_{\pi^{1 / p^{n}}, T}\right)^{\smile} .
$$

So it suffices to show that

$$
\left(\operatorname{tr}_{\pi^{1 / p^{n}}, T}\right)^{\smile}(M)=\left\{z \in M: \pi^{1 / p^{n}} \otimes z=0 \in T \otimes_{R} M\right\} .
$$

By Theorem 8.18, we have

$$
\left(\operatorname{tr}_{\pi^{1 / p^{n}}, T}\right)^{\smile}=\operatorname{tom}_{\pi^{1 / p^{n}, T}} .
$$

By Proposition 8.17, this gives us the desired result.

Remark 11.4. With cl as above, $\operatorname{ann}_{R}\left(0_{E}^{\mathrm{cl}}\right)=\lim _{\longrightarrow} \operatorname{tr}_{\pi^{1 / p^{n}}, T}(R)$ by Theorem 5.5 .

In [PR20], Pérez and the second named author studied this closure operation in the context of almost balanced big Cohen-Macaulay algebras.

Definition 11.5 ([And18]). $T$ is an almost (balanced) big Cohen-Macaulay algebra with respect to $\pi^{1 / p^{\infty}}$ if $T / m T$ is not almost 0 with respect to $\pi^{1 / p^{\infty}}$ (i.e., it is not the case that $\pi^{1 / p^{n}} T / m T=0$ for all $n \gg 0$ ), and for every system of parameters $x_{1}, \ldots, x_{d}$ on $R$,

$$
\pi^{1 / p^{n}} \cdot \frac{\left(x_{1}, \ldots, x_{i}\right):_{T} x_{i+1}}{\left(x_{1}, \ldots, x_{i}\right)}=0
$$

for all $n>0,0 \leq i \leq d-1$.

The following result now follows immediately from Proposition 11.3 .

Theorem 11.6 ([PR20, Theorem 6.5]). Let $R$ be a complete local domain, $c l$ be as above, and additionally assume that $T$ is p-torsion free, $\pi$ is a nonzerodivisor, and $T$ is an almost balanced big Cohen-Macaulay algebra. Then

$$
\operatorname{ann}_{R}\left(0_{E}^{\mathrm{cl}}\right)=\sum_{n>0} \sum_{\psi: T \rightarrow R} \psi\left(\pi^{1 / p^{n}} T\right)=\sum_{n>0} \operatorname{im}\left(T^{*} \otimes \pi^{1 / p^{n}} T \rightarrow R\right),
$$

where the map sends $\psi \otimes \pi^{1 / p^{n}} t \mapsto \psi\left(\pi^{1 / p^{n}} t\right)$. 


\section{Good localization is DUAL to GOOD COLOCALization}

In this section, $R$ is a commutative Noetherian ring with identity.

We introduce the following concept, with notation and terminology due to Richardson:

Definition 12.1 (Ric06 $)$. Let $(R, \mathfrak{m})$ be a Noetherian local ring, $\mathfrak{p} \in \operatorname{Spec} R$, and $M$ an $R$-module. Then the (Richardson) colocalization of $M$ with respect to $\mathfrak{p}$ is the $\widehat{R_{\mathfrak{p}}}$-module

$$
{ }^{\mathfrak{p}} M:=\operatorname{Hom}_{R}\left(\operatorname{Hom}_{R}\left(M, E_{R}(R / \mathfrak{m})\right), E_{R}(R / \mathfrak{p})\right) .
$$

This concept had earlier been explored (though without an explicit name, and with different notation (namely $M^{\vee \vee_{\mathfrak{p}}}$ ) by K. Smith Smi94, Smi95. We start by recalling the following useful principle.

Lemma 12.2 ([Smi94, Lemma 3.1(iv)]). Let $(R, \mathfrak{m})$ be a Noetherian local ring, let $E=E_{R}(R / \mathfrak{m})$, and let $M$ be an $R$-submodule of $E$. Then

$$
M^{\vee} \cong \hat{R} / \operatorname{ann}_{\hat{R}}(M)
$$

Note that when $M$ is an artinian $R$-module, ${ }^{\mathfrak{p}} M$ is an artinian $\widehat{R_{\mathfrak{p}}}-$ module Ric06, Theorem 2.3]. Also note that ${ }^{\mathfrak{p}}(-)$ is an exact covariant functor on the category of $R$-modules, as it consists of applying Hom into an injective $R$-module twice. We have the following:

Theorem 12.3. Let $(R, \mathfrak{m})$ be a complete Noetherian local ring, and let $\mathfrak{p} \in \operatorname{Spec} R$. Let $\sigma$ be a submodule selector on Artinian $R$-modules and $\sigma^{\prime}$ a submodule selector on artinian $\widehat{R_{\mathfrak{p}}}$-modules. Let $\tau=\sigma^{-}$(over $R$ ) and $\tau^{\prime}=\left(\sigma^{\prime}\right)^{\smile}$ (over $\left.\widehat{R_{\mathfrak{p}}}\right)$. Then $\tau^{\prime}\left(\widehat{N_{\mathfrak{p}}}\right)=\widehat{\tau(N)_{\mathfrak{p}}}$ for all finitely generated $R$ modules $N$ if and only if $\sigma^{\prime}\left({ }^{\mathfrak{p}} M\right)={ }^{\mathfrak{p}} \sigma(M)$ for all Artinian $R$-modules $M$.

Proof. Let ${ }^{\vee^{\prime}}$ denote the Matlis dual operation over the ring $\widehat{R_{\mathfrak{p}}}$. That is, if $U$ is an $\widehat{R_{\mathfrak{p}}}$-module, then $U^{\vee^{\prime}}:=\operatorname{Hom}_{\widehat{R_{\mathfrak{p}}}}\left(U, E_{R}(R / \mathfrak{p})\right)$.

First suppose $\tau^{\prime}\left(\widehat{N_{\mathfrak{p}}}\right)=\widehat{\tau(N)_{\mathfrak{p}}}$ for all finitely generated $R$-modules $N$. Let $M$ be an Artinian $R$-module. Then

$$
\begin{aligned}
{ }^{\mathfrak{p}} \sigma(M) & =\left(\sigma(M)^{\vee} \otimes_{R} \widehat{R_{\mathfrak{p}}}\right)^{\vee^{\prime}}=\left(\tau^{\smile}(M)^{\vee} \otimes_{R} \widehat{R_{\mathfrak{p}}}\right)^{\vee^{\prime}} \\
& =\left(\frac{M^{\vee}}{\tau\left(M^{\vee}\right)} \otimes_{R} \widehat{R_{\mathfrak{p}}}\right)^{\vee^{\prime}} \cong\left(\frac{\widehat{\left(M^{\vee}\right)_{\mathfrak{p}}}}{\tau \widehat{\tau\left(M^{\vee}\right)_{\mathfrak{p}}}}\right)^{\vee^{\prime}} \\
& =\left(\tau^{\prime}\right)^{\smile}\left(\overline{\left(M^{\vee}\right)_{\mathfrak{p}}} \vee^{\prime}\right)=\sigma^{\prime}\left({ }^{\mathfrak{p}} M\right) .
\end{aligned}
$$


Conversely, suppose $\sigma^{\prime}\left({ }^{\mathfrak{p}} M\right)={ }^{\mathfrak{p}} \sigma(M)$ for all Artinian $R$-modules $M$. Let $N$ be a finitely generated $R$-module. Then

$$
\begin{aligned}
& \tau^{\prime}\left(\widehat{N_{\mathfrak{p}}}\right)=\left(\sigma^{\prime}\right)^{\smile}\left(\widehat{N_{\mathfrak{p}}}\right)=\left(\frac{\left(\widehat{N_{\mathfrak{p}}}\right)^{\vee^{\prime}}}{\sigma^{\prime}\left(\left(\widehat{N_{\mathfrak{p}}}\right)^{\vee^{\prime}}\right)}\right)^{\vee^{\prime}}=\left(\frac{{ }^{\mathfrak{p}}\left(N^{\vee}\right)}{\sigma^{\prime}\left(\mathfrak{p}\left(N^{\vee}\right)\right)}\right)^{\vee^{\prime}} \\
& =\left(\frac{\mathfrak{p}\left(N^{\vee}\right)}{\mathfrak{p} \sigma\left(N^{\vee}\right)}\right)^{\vee^{\prime}} \cong\left(\mathfrak{p}\left(\frac{N^{\vee}}{\sigma\left(N^{\vee}\right)}\right)\right)^{\vee^{\prime}}=\left({ }^{\mathfrak{p}}\left(\sigma^{\smile}(N)^{\vee}\right)\right)^{\vee^{\prime}} \\
& ={\bar{\sigma}(N)_{\mathfrak{p}}}^{=} \overline{\tau(N)_{\mathfrak{p}}}
\end{aligned}
$$

In particular, we have:

Theorem 12.4. Let $R$ be a complete Noetherian reduced F-finite ring of prime characteristic $p>0$. Let $M$ be an artinian $R$-module. Then for any prime ideal $\mathfrak{p}$, we have ${ }^{\mathfrak{p}}\left(0_{M}^{*}\right)=0_{\mathfrak{p}}^{*}$, i.e., tight closure co-localizes.

Proof. By Proposition 10.2 (2), tight interior agrees with the dual of tight closure in the sense of this paper. Recall that in this circumstance, tight interior localizes and commutes with completion [ES14, Corollary 2.11]. That is, for any finitely generated $R$-module $N$, we $\tau^{\prime}\left(\widehat{N_{\mathfrak{p}}}\right)=\widehat{\tau(N)_{\mathfrak{p}}}$, where $\tau$ is tight interior on $R$-modules and $\tau^{\prime}$ is tight interior on $\widehat{R_{\mathfrak{p}}}$-modules. Then setting $\sigma=\tau^{\sim}$ to be the submodule selector $\sigma(M)=0_{M}^{*}$, we apply Theorem 12.3 to get the result.

As a special case, we recover the following fact, implicit in [LS01] as evidenced by the second proof below.

Corollary 12.5. Let $R$ be a complete Noetherian reduced $F$-finite ring of prime characteristic $p>0$. Let $E$ be the injective hull of the residue field of $R$. Let $\mathfrak{p} \in \operatorname{Spec} R$. Then

$$
0_{E_{R}(R / \mathfrak{p})}^{*}={ }^{\mathfrak{p}}\left(0_{E}^{*}\right)
$$

Proof 1, via new methods. By definition of $\mathfrak{p}(-)$ and since $E^{\vee}=R$, we have ${ }^{\mathfrak{p}} E=E_{R}(R / \mathfrak{p})$. The result then follows from Theorem 12.4 with $E=M$.

Proof 2, via old methods. We have the following chain of equalities:

$$
\begin{array}{rlr}
0_{E_{R}(R / \mathfrak{p})}^{*} & \operatorname{Hom}_{\widehat{R_{\mathfrak{p}}}}\left(\widehat{R_{\mathfrak{p}}} / \operatorname{ann}_{\widehat{R_{\mathfrak{p}}}}\left(0_{E_{R}(R / \mathfrak{p})}^{*}\right), E_{R}(R / \mathfrak{p})\right) & \text { by Lemma } 12.2 \\
& =\operatorname{Hom}_{\widehat{R_{\mathfrak{p}}}}\left(\widehat{R_{\mathfrak{p}}} / \operatorname{ann}_{R}\left(0_{E}^{*} \widehat{R_{\mathfrak{p}}}, E_{R}(R / \mathfrak{p})\right)\right. & \text { by [LS01, Theorem } 7.1] \\
& =\operatorname{Hom}_{\widehat{R_{\mathfrak{p}}}}\left(\widehat{R_{\mathfrak{p}}} \otimes_{R} R / \operatorname{ann}_{R}\left(0_{E}^{*}\right), E_{R}(R / \mathfrak{p})\right) & \text { by flatness of } \widehat{R_{\mathfrak{p}}} \text { over } R \\
& \cong \operatorname{Hom}_{R}\left(R / \operatorname{ann}_{R}\left(0_{E}^{*}\right), \operatorname{Hom}_{\widehat{R_{\mathfrak{p}}}}\left(\widehat{R_{\mathfrak{p}}}, E_{R}(R / \mathfrak{p})\right)\right) & \text { by Hom- } \otimes \text { adjunction } \\
& \cong \operatorname{Hom}_{R}\left(R / \operatorname{ann}_{R}\left(0_{E}^{*}\right), E_{R}(R / \mathfrak{p})\right) & \\
& =\operatorname{Hom}_{R}\left(\left(0_{E}^{*}\right)^{\vee}, E_{R}(R / \mathfrak{p})\right) & \text { by Lemma } 12.2 \\
& ={ }^{\mathfrak{p}}\left(0_{E}^{*}\right) &
\end{array}
$$

We also get the following as a corollary: 
Theorem 12.6. Let $R$ be a complete Noetherian local ring and $B$ a finitely generated $R$-module. Let $M$ be an artinian $R$-module. Then

$$
\mathfrak{p}\left(0_{M}^{\mathrm{cl}_{B}}\right)=0_{\mathfrak{p}}^{\mathrm{cl}_{\widehat{B p}}}
$$

In particular, we have

$$
\mathfrak{p}\left(0_{E}^{\mathrm{cl}_{B}}\right)=0_{E_{R}(R / \mathfrak{p})}^{\mathrm{cl}_{\widehat{B \mathfrak{p}}}} .
$$

Proof. This follows from Theorems 12.3, 8.12, and 8.18.

Finally, we observe a curious relationship between $I$-adic completion, $\mathfrak{p}$ colocalization, and $\mathfrak{p}$-adic completion:

Theorem 12.7. Let $R$ be a complete Noetherian local ring, let $\mathfrak{p} \in \operatorname{Spec} R$, and $M$ an artinian $R$-module. Then

$$
\bigcap_{n \geq 0}\left(\left(\widehat{I R_{\mathfrak{p}}}\right)^{n} \cdot \mathfrak{p} M\right)=\mathfrak{p}\left(\bigcap_{n \geq 0}\left(I^{n} M\right)\right) .
$$

That is, the colocalization at $\mathfrak{p}$ of the kernel of the I-adic completion map on $M$ is the same as the kernel of the $\widehat{I R_{\mathfrak{p}}}$-adic completion map on the colocalization of $M$ at $\mathfrak{p}$.

Specializing to the case $M=E$, we have

$$
\bigcap_{n \geq 0}\left(\left(\widehat{I R_{\mathfrak{p}}}\right)^{n} \cdot E_{R}(R / \mathfrak{p})\right)=\mathfrak{p}\left(\bigcap_{n \geq 0}\left(I^{n} E\right)\right) .
$$

Proof. Let $\sigma$ be the submodule selector on $R$-modules given by $M \mapsto \bigcap_{n} I^{n} M$. Then by Corollary 8.23, $\tau=\sigma^{\smile}=H_{I}^{0}$. Similarly, letting $\sigma^{\prime}$ be the submodule selector on $\widehat{R_{\mathfrak{p}}}$-modules given by $M \mapsto \bigcap_{n}\left(\widehat{I R_{\mathfrak{p}}}\right)^{n} M$, we have $\tau^{\prime}=\left(\sigma^{\prime}\right)^{\smile}=$ $H_{\overline{I R_{\mathfrak{p}}}}^{0}$. Since local cohomology commutes with flat base change [BS98, 4.3.2 Flat Base Change Theorem], and since completion of a finitely generated module coincides with tensoring with the completed ring, we have

$$
\begin{aligned}
\tau^{\prime}\left(\widehat{N_{\mathfrak{p}}}\right) & =H_{\overline{I R_{\mathfrak{p}}}}^{0}\left(\widehat{N_{\mathfrak{p}}}\right)=H_{I \widehat{R_{\mathfrak{p}}}}^{0}\left(N \otimes_{R} \widehat{R_{\mathfrak{p}}}\right) \\
& =H_{I}^{0}(N) \otimes_{R} \widehat{R_{\mathfrak{p}}}=\widehat{H_{I}^{0}(N)_{\mathfrak{p}}}=\widehat{\tau(N)_{\mathfrak{p}}}
\end{aligned}
$$

for all finitely generated $R$-modules $N$. Thus, by Theorem 12.3 , we have for all Artinian $R$-modules $M$ that

$$
\bigcap_{n \geq 0}\left(\left(\widehat{I R_{\mathfrak{p}}}\right)^{n} \cdot{ }^{\mathfrak{p}} M\right)=\sigma^{\prime}\left({ }^{\mathfrak{p}} M\right)={ }^{\mathfrak{p}} \sigma(M)={ }^{\mathfrak{p}}\left(\bigcap_{n \geq 0}\left(I^{n} M\right)\right) .
$$

The final statement follows from the fact that ${ }^{\mathfrak{p}} E=E_{R}(R / \mathfrak{p})$.

\section{ACKNOWLEDGMENT}

We are grateful for suggestions from Geoffrey Dietz, Jesse Elliott, Haydee Lindo, Karen Smith, Janet Vassilev, and the anonymous referee, all of which improved the paper. 


\section{REFERENCES}

[And18] Yves André, La conjecture du facteur direct, Publ. Math. Inst. Hautes Études Sci. 127 (2018), 71-93. MR 3814651

[BM10] Holger Brenner and Paul Monsky, Tight closure does not commute with localization, Ann. of Math. (2) 171 (2010), no. 1, 571-588.

[BS98] Markus P. Brodmann and Rodney Y. Sharp, Local cohomology: an algebraic introduction with geometric applications, Cambridge Studies in Advanced Mathematics, vol. 60, Cambridge Univ. Press, Cambridge, 1998.

[CLVW06] John Clark, Christian Lomp, Narayanaswami Vanaja, and Robert Wisbauer, Lifting modules, Frontiers in Mathematics, Birkhäuser Verlag, Basel, 2006.

[Die10] Geoffrey D. Dietz, A characterization of closure operations that induce big Cohen-Macaulay modules, Proc. Amer. Math. Soc. 138 (2010), no. 11, 38493862 .

[DT95] Dikran Dikranjan and Walter Tholen, Categorical structure of closure operators, Mathematics and its Applications, vol. 346, Kluwer, Dordrecht, 1995.

[EG89] E. Graham Evans, Jr. and Phillip A. Griffith, Order ideals, Commutative algebra (Berkeley, CA, 1987), Math. Sci. Res. Inst. Publ., vol. 15, Springer, New York, 1989, pp. 213-225. MR 1015519

[EJ00] Edgar E. Enochs and Overtoun M. G. Jenda, Relative homological algebra, De Gruyter Expositions in Mathematics, vol. 30, Walter de Gruyter \& Co., Berlin, 2000 .

[Eps12] Neil Epstein, A guide to closure operations in commutative algebra, Progress in Commutative Algebra 2 (Berlin/Boston) (Christopher Francisco, Lee Klingler, Sean Sather-Wagstaff, and Janet C. Vassilev, eds.), De Gruyter Proceedings in Mathematics, De Gruyter, 2012, pp. 1-37.

[ES14] Neil Epstein and Karl Schwede, A dual to tight closure theory, Nagoya Math. J. 213 (2014), 41-75.

[EU] Neil Epstein and Bernd Ulrich, Liftable integral closure, arXiv:1309.6966 [math.AC], to appear in J. Commut. Algebra.

[EY12] Neil Epstein and Yongwei Yao, Criteria for flatness and injectivity, Math. Z. 271 (2012), no. 3-4, 1193-1210.

[HH90] Melvin Hochster and Craig Huneke, Tight closure, invariant theory, and the Briançon-Skoda theorem, J. Amer. Math. Soc. 3 (1990), no. 1, 31-116.

[Hoc07] Melvin Hochster, Foundations of Tight Closure Theory, http://www.math.1sa.umich.edu/ hochster/711F07/fndtc.pdf, 2007.

[HS06] Craig Huneke and Irena Swanson, Integral closure of ideals, rings, and modules, London Math. Soc. Lecture Note Ser., vol. 336, Cambridge Univ. Press, Cambridge, 2006.

[HT04] Nobuo Hara and Shunsuke Takagi, On a generalization of test ideals, Nagoya Math. J. 175 (2004), 59-74.

[Hun96] Craig Huneke, Tight closure and its applications, CBMS Reg. Conf. Ser. in Math., vol. 88, Amer. Math. Soc., Providence, RI, 1996.

[Lam99] T. Y. Lam, Lectures on modules and rings, Graduate Texts in Mathematics, vol. 189, Springer-Verlag, New York, 1999. MR 1653294

[Lin17] Haydee Lindo, Trace ideals and centers of endomorphism rings of modules over commutative rings, J. Algebra 482 (2017), 102-130. MR 3646286

[LS01] Gennady Lyubeznik and Karen E. Smith, On the commutation of the test ideal with localization and completion, Trans. Amer. Math. Soc. 353 (2001), no. 8, 3149-3180. MR 1828602

[PR20] Felipe Pérez and Rebecca R.G., Characteristic-free test ideals, arXiv:1907.02150, to appear in Trans. Amer. Math. Soc., 2020. 
[R.G16] Rebecca R.G., Closure operations that induce big Cohen-Macaulay modules and classification of singularities, J. Algebra 467 (2016), 237-267.

[Ric06] Andrew S. Richardson, Co-localization, co-support and local homology, Rocky Mountain J. Math. 36 (2006), no. 5, 1679-1703.

[Smi94] Karen E. Smith, Tight closure of parameter ideals, Invent. Math. 115 (1994), no. 1, 41-60.

[Smi95] Test ideals in local rings, Trans. Amer. Math. Soc. 347 (1995), no. 9, $3453-3472$.

Department of Mathematical Sciences, George Mason University, Fairfax, VA 22030

Email address: nepstei2@gmu.edu

Department of Mathematical Sciences, George Mason University, Fairfax, VA 22030

Email address: rrebhuhn@gmu.edu 